\title{
A Closer Look at Multiple Forking: Leveraging (In)dependence for a Tighter Bound
}

\author{
Sanjit Chatterjee and Chethan Kamath, \\ Indian Institute of Science, Bangalore. \\ \{sanjit, chethan0510\}@csa.iisc.ernet.in \\ January 1, 2014
}

\begin{abstract}
Boldyreva et al. introduced the notion of multiple forking (MF) as an extension of (general) forking to accommodate nested oracle replay attacks. The primary objective of a (multiple) forking algorithm is to separate out the oracle replay attack from the actual simulation of protocol to the adversary, and this is achieved through the intermediary of a so-called wrapper algorithm. Multiple forking has turned out to be a useful tool in the security argument of several cryptographic protocols. However, a reduction employing the MF Algorithm incurs a significant degradation of $\mathrm{O}\left(q^{2 n}\right)$, where $q$ denotes the upper bound on the underlying random oracle calls and $n$, the number of forkings.

In this work we take a closer look at the reasons for the degradation with a tighter security bound in mind. We nail down the exact set of conditions for the success of the MF Algorithm. A careful analysis of the protocols (and corresponding security argument) employing multiple forking allow us to relax the overly restrictive conditions of the original MF Algorithm. To achieve this, we club two consecutive invocations of the underlying wrapper into a single logical unit of wrapper $\mathcal{Z}$. We then use $\mathcal{Z}$ to formulate the notion of "dependence" and "independence" among different rounds of the wrapper in the MF Algorithm. The (in)dependence conditions lead to a general framework for multiple forking and significantly better bound for the MF Algorithm. Leveraging (in)dependence to the full reduces the degradation from $\mathrm{O}\left(q^{2 n}\right)$ to $\mathrm{O}\left(q^{n}\right)$. By implication, the cost of a forking involving two random oracles (augmented forking) matches that involving a single random oracle (elementary forking).
\end{abstract}

Finally, we study the effect of these observations on the security of the existing schemes. We conclude that by careful design of the protocol (and the wrapper in the security reduction) it is possible to harness our observations to the full extent.

Keywords: Oracle Replay Attack, Forking Lemma, Multiple-Forking Lemma, Provable Security, Tightness. 


\section{Contents}

1 Introduction 3

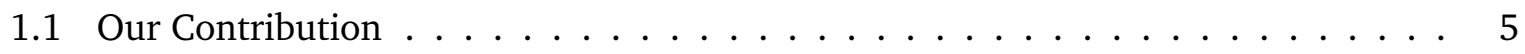

2 Multiple-Forking: A Closer Look $\quad 6$

2.1 Tightness: An Intuitive Picture . . . . . . . . . . . . . . . . . . . 8

2.2 Road-map to a Better Analysis . . . . . . . . . . . . . . . . . . . . . 9

2.3 A General Multiple-Forking Algorithm . . . . . . . . . . . . . . . . . . . . . 11

3 Harnessing (In)Dependence $\quad 12$

3.1 Multiple-Forking with Index (In)Dependence $\ldots \ldots \ldots \ldots \ldots$

4 Revisiting the Security Argument of Existing Protocols 17

4.1 Random-Oracle Dependence . . . . . . . . . . . . . . . . . . 17

4.2 The Boldyreva-Palacio-Warinschi Proxy Signature Scheme . . . . . . . . . . . . 19

4.2 .1 Improved Security Argument . . . . . . . . . . . . . . . . . . 20

4.3 The Galindo-Garcia Identity-Based Signature . . . . . . . . . . . . . . 20

4.3.1 Modified Galindo-Garcia IBS . . . . . . . . . . . . . . . . . 21

4.4 The Chow-Ma-Weng ZKP for Simultaneous Discrete Logarithms . . . . . . . . . . 21

4.4 .1 A Case for (In)Dependence . . . . . . . . . . . . . . . . 22

4.4 .2 Improved Argument . . . . . . . . . . . . . . . . . 23

5 Conclusion $\quad 24$

$\begin{array}{ll}\text { A General Forking } & 26\end{array}$

B (Original) Multiple-Forking Algorithm 27

C Harnessing (In)Dependence $\quad 28$

C.1 Detailed Steps for Lemma $2 \ldots \ldots \ldots$

C.2 Multiple-Forking with Index Independence . . . . . . . . . . . . . . 30

C.3 Multiple-Forking with Index Dependence . . . . . . . . . . . . . . . . . . 32

D Constructions $\quad 35$

D.1 The Boldyreva-Palacio-Warinschi Proxy Signature Scheme . . . . . . . . . . 35

D.2 The (Original) Galindo Garcia IBS . . . . . . . . . . . . . . . . 37

D.3 The Modified Galindo Garcia IBS . . . . . . . . . . . . . . . . 37

D.4 Chow-Ma-Weng Zero-Knowledge Argument $\ldots \ldots \ldots \ldots \ldots$

E Security Argument for Modified Galindo-Garcia IBS 38

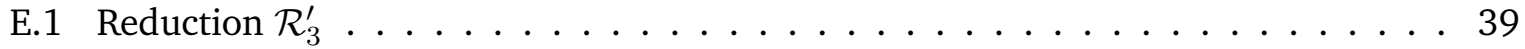

E.1.1 Analysis. . . . . . . . . . . . . . . . . . . 40 


\section{Introduction}

The machinery of oracle replay attack of Pointcheval and Stern [PS00] plays a pivotal role in the security argument of a large class ${ }^{1}$ of signature schemes [ElG85, Sch91, Oka93]. In the elementary version of replay attack ${ }^{2}$, the simulator runs the adversary twice on related inputs in order to solve the underlying hard problem. The probability of success of the replay attack is then bounded by the Forking Lemma [PS00]. Bellare and Neven [BN06], however, observed that the "Forking Lemma is something purely probabilistic, not about signatures" and proposed a more abstract version called the General Forking (GF) Lemma. The concept of general forking is formulated in terms of randomised algorithms and its outputs, leaving out the notions of signature scheme as well as random oracles [BR93] altogether. The claimed advantage is to allow for more modular and easy to verify proof of cryptographic schemes that apply the notion of forking in their security argument.

Multiple forking. The concept of forking was further generalised by Boldyreva et al. leading to the Multiple Forking (MF) Algorithm [BPW12]. The immediate motivation behind this new abstraction was to argue the security of a proxy signature scheme that uses more than one hash function (modelled as random oracles). The MF Algorithm allows one to mount the so-called nested replay attacks by rewinding the adversary several times on related inputs. In particular, a nested oracle replay attack involves multiple augmented forkings ${ }^{3}$. The MF Algorithm retains the modularity advantage of GF Algorithm and has been applied in several other security arguments [GG09, CMW12, CKK13] in a more-or-less black-box fashion. Note that the generalisation of forking due to Bagherzhandi et al. [BCJ08] is different from that in [BPW12].

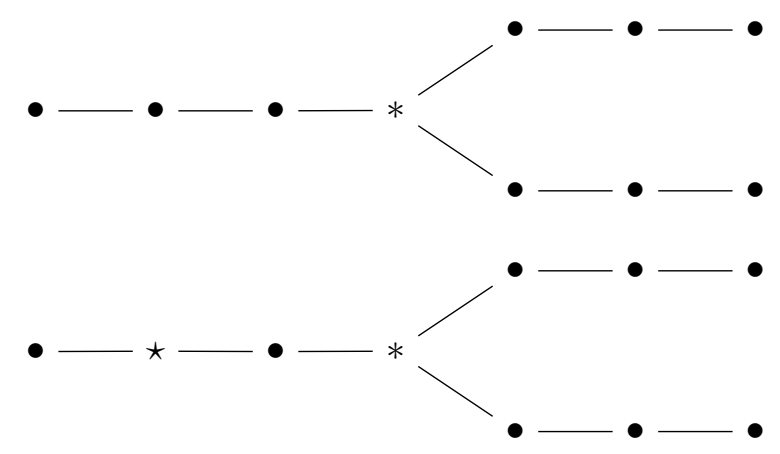

Figure 1: Elementary forking (top) vs. augmented forking (bottom): Elementary forking is successful if the target indices $(*)$ for the two rounds match. Augmented forking involves two random oracles and, hence, to be successful, the additional target indices $(\star)$ also should match.

The modularity of the (General/Multiple) Forking Lemma allows one to abstract out the probabilistic analysis of the rewinding process from the actual simulation in the security argument. The gap between the abstract and the concrete is, then, bridged using the so-called "wrapper" algorithm. While the GF/MF Algorithm takes care of the replay attack, it is the wrapper that handles the simulation of the protocol environment to the actual adversary. The reduction consists of invoking the appropriate forking algorithm (on the associated wrapper) and utilising its outputs to solve the underlying hard problem. So the design of the wrapper is central to any security argument involving GF/MF Algorithm. In fact, the design depends

\footnotetext{
${ }^{1}$ To be precise, the signature schemes obtained from three-round identification schemes ( $\Sigma$-protocols) through the Fiat-Shamir transformation [FS87].

${ }^{2}$ We will use the terms forking and oracle replay attack interchangeably.

${ }^{3}$ We clearly distinguish "augmented" forking from "elementary" forking: the former involves replay of two random oracles whereas the latter, only one random oracle. Henceforth, whenever we refer to multiple forkings, we are implicitly referring to multiple "augmented" forking.
} 
on the actual protocol and the security model used-see, e.g., [BN06, BPW12] for the concrete design of the wrappers in their respective contexts.

Role of the wrapper. Let's now take a simplistic ${ }^{4}$ look at how the GF Algorithm (given in Appendix A), together with the wrapper $\mathcal{Y}$, is used to launch the elementary replay attack. The input to $\mathcal{Y}$ consists of some external randomness $\left(s_{1}, \ldots, s_{q}\right)$ and the internal coins $(\rho)$ for adversary; the output, whereas, consists of an index $I$.

Consider the first invocation of $\mathcal{Y}$ (on $s_{1}, \ldots, s_{q} ; \rho$ ) within the GF Algorithm: $\mathcal{Y}$ simulates the protocol environment to the actual adversary $\mathcal{A}$ having access to $\rho$. $\mathcal{Y}$ responds to the random oracle queries of $\mathcal{A}$ using $s_{1}, \ldots, s_{q}$. At the end of the simulation, $\mathcal{Y}$ outputs an index $I$ that refers to the target query ${ }^{5}$; if the adversary did not make the target query, $I$ is set to 0 (indicating failure). Next, the GF Algorithm invokes $\mathcal{Y}$ on an input that is related to the first invocation $\left(s_{1}, \ldots, s_{I-1}, s_{I}^{\prime}, \ldots, s_{q}^{\prime} ; \rho\right)$. The behaviour of $\mathcal{A}$ remains identical to the first round of simulation, right up to the $I^{\text {th }}$ random oracle query, at which point it diverges (assuming $\left.s_{I}^{\prime} \neq s_{I}\right)$. This is tantamount to forking $\mathcal{A}$ at the index $I$. The forking is successful, if the target index for the second round of simulation is the same as that for the first, i.e., $I^{\prime}=I$. One can clearly see how the wrapper acts as an intermediary between the abstract GF Algorithm and the adversary in the concrete setting of the reduction.

While the role of wrapper remains the same in the MF Algorithm, there are a few significant changes in its actual structure. The wrapper now simulates two random oracles and hence its output contains a pair of indices $(I, J)$ with $J<I$. The two indices are usually associated to the target queries made to the two random oracles involved in the augmented replay attack. For reductions employing the MF Algorithm, the design of the wrapper becomes a bit more involved because of the additional index in its output. In particular, the relative "order" among the two target oracle calls must be taken into consideration in the design of the wrapper. (We'll later see how neglecting the order of the indices, or even worse, using the MF Algorithm as a black-box may lead the reductions to fail.) As the name suggests, the MF Algorithm allows the possibility of more than one forking-this adds another level of complexity in the overall structure.

The cost of multiple forking. The Forking Lemma gives us a lower bound on the probability of success of the forking algorithm in terms of the success probability of the associated wrapper (and hence, the underlying adversary). Roughly speaking, the cost of forking can be measured in terms of the degradation incurred in the forking process. Let $q$ denote the upper bound on the number of random oracle queries, then the cost of general forking is roughly $\mathrm{O}(q)$ (and evidence suggests that the bound is more or less optimal [Seu12]). As for multiple forking, the cost according to the MF Lemma (see Lemma 8 in Appendix B), is roughly $\mathrm{O}\left(q^{2 n}\right)$, where $q$ is the sum of the upper bound on the queries to the random oracles involved and $n$ is, loosely speaking, the number of forking (so the wrapper is called $n+1$ times). ${ }^{6}$ Consequently, the cost of single augmented forking is $\mathrm{O}\left(q^{2}\right)$ (even though there is only one forking involved). As we see, the bound is quite loose and naturally, the protocols employing the MF Lemma for their security suffer from this bound. This is indicated in the concluding statement of [BPW12] where the authors mention that the concrete security bound of their scheme is not particularly tight and they leave the possibility of a tighter reduction as an open question. In fact, for all security reductions that employ the MF Lemma [BPW12, GG09, CMW12, CKK13], the degradation primarily stems from the loose lower bound of the lemma. Hence, it's important to ask whether

\footnotetext{
${ }^{4}$ For the time being, let's not consider the input string $x$ or the side-output $\sigma$.

${ }^{5}$ The random oracle query that is used by $\mathcal{A}$ to produce its desired output is termed the target query and the index of this query is the target index.

${ }^{6}$ To be precise, if acc denotes the probability with which the wrapper is successful for one round, then the cost of general forking is $\mathrm{O}(q / a c c)$ and that of multiple forking is $\mathrm{O}\left(q^{2 n} / a c c^{n}\right)$. We ignore the acc factor in the discussion here.
} 
and to what extent it is possible to improve upon this bound. Investigating these questions in the concrete context of cryptographic protocols forms the focal point of this work.

\subsection{Our Contribution}

Multiple forking is a further generalisation of general forking and once formulated, this abstract notion has been applied in the analysis of concrete cryptographic protocols essentially in a black-box way. Here we undertake a journey from the concrete to the abstract. This has two (complementary) parts. We take a critical look at the various conditions that decide the success of the MF Algorithm as well as revisit the concrete security arguments employing the MF Lemma. This study allows us to come up with a better abstraction of multiple forking.

By the very nature of the rewinding mechanism, the wrapper algorithm is always invoked in pairs. Based on this simple observation, we club two consecutive invocations of the wrapper into a single logical unit of wrapper $\mathcal{Z}$. Intuitively, wrapper $\mathcal{Z}$ can be viewed as one invocation of the GF Algorithm of [BN06]. Utilizing the extra level of modularity provided by $\mathcal{Z}$, we nail down the exact conditions for the success of multiple forking. This, coupled with our investigation of the actual security arguments employing MF Lemma allow us to formulate two crucial observations: called, respectively, the "independence" condition $\left(\mathrm{O}_{\mathrm{I}}\right)$ and the "dependence" condition $\left(\mathrm{O}_{\mathrm{D}}\right)$. $\mathrm{O}_{\mathrm{I}}$ is formulated by a careful abstraction of the exact requirements in the security reductions. In short, it allows the relaxation of success condition related to the index $I$ across the logical wrapper $\mathcal{Z}$. $\mathrm{O}_{\mathrm{D}}$, on the other hand, has its root in the notion of hash function dependence, which is actually observed in the cryptographic protocols.

A general framework. Based on the above observations, we propose a general framework for the application of the MF Algorithm, which we call the General Multiple-Forking (GMF) Algorithm, and an associated GMF Lemma. Our framework captures the original MF as well as the observations $\mathrm{O}_{\mathrm{I}}$ and $\mathrm{O}_{\mathrm{D}}$ (separately and together) leading to four different versions of the lemma. We prove the new versions of the lemma. Naturally, the analysis becomes more involved as we incorporate the above two conditions-the most involved case occurs when one captures both the observations $\mathrm{O}_{I}$ and $\mathrm{O}_{D}\left(\mathrm{O}_{\{1, \mathrm{D}\}}\right.$, in short). We draw from existing techniques [BN06, BPW12] as well as introduce some new optimisations to significantly improve upon the existing bound. To be exact, the degradation reduces from $\mathrm{O}\left(q^{2 n}\right)$ to $\mathrm{O}\left(q^{n}\right)$ when both the observations are incorporated in the analysis (see Table 2 for a summary). Thus, informally, we have:

Main Result (see Lemma 2). By carefully designing the protocol, multiple forking can be carried out with a success probability of $\Omega\left(\epsilon^{n+1} / q^{n}\right)$.

Corollary (see Claim 1, Remark 4). A single augmented forking can be launched as efficiently as an elementary forking.

Recall that the GF Algorithm of [BN06] captures a single elementary forking with a degradation of $\mathrm{O}(q)$. Our MF Algorithm with $\mathrm{O}_{\{\mathrm{I}, \mathrm{D}\}}$, according to the above corollary, seems to be the best possible generalisation of general forking.

Effect on cryptographic schemes. Finally, we study the applicability of the observations on the security of the existing schemes that employ multiple forking [BPW12, GG09, CMW12]. We conclude that by careful design of the protocol (or, for that matter, by easily modifying existing protocols) it is possible to harness both the observations to the full extent. Thus, we end up with tighter security arguments for the protocols and under the same (hardness) assumptions (see Table 1). In addition, the notion of random-oracle dependence may be of independent interest as it is handy in certain situations other than multiple forking [YZ13, YADV $\left.{ }^{+} 12\right]$. 
On a related note, we also emphasise on the importance of the proper design of the wrapper algorithm taking into account the intricacies of the actual security argument.

\begin{tabular}{|c|c|c|}
\hline \multirow{2}{*}{ Protocol } & \multicolumn{2}{|c|}{ Degradation } \\
\cline { 2 - 3 } & Before & After \\
\hline [BPW12] & $\mathrm{O}\left(q^{10} / \epsilon^{5}\right)$ & $\mathrm{O}\left(q^{5} / \epsilon^{5}\right)$ \\
\hline [GG09] & $\mathrm{O}\left(q^{6} / \epsilon^{3}\right)$ & $\mathrm{O}\left(q^{3} / \epsilon^{3}\right)$ \\
\hline [CMW12] & $\mathrm{O}\left(q^{10} / \epsilon^{5}\right)$ & $\mathrm{O}\left(q^{5} / \epsilon^{5}\right)$ \\
\hline
\end{tabular}

Table 1: Comparison of the security degradation for protocols before and after our result. $q$ denotes the upper bound on the respective hash oracle queries; $\epsilon$ is the advantage that the adversary has in the respective protocols.

Notations. We adopt the notations commonly used in the literature. $s \stackrel{U}{\leftarrow} \mathbb{S}$ denotes picking an element $s$ uniformly at random from the set $\mathbb{S}$. In general, $\left\{s_{1}, \ldots, s_{n}\right\} \stackrel{\cup}{\leftarrow} \mathbb{S}$ denotes picking elements $s_{1}, \ldots, s_{n}$ independently and uniformly at random from the set $\mathbb{S}$. In a similar manner, $s \stackrel{\$}{\leftarrow} \mathbb{S}$ and $\left\{s_{1}, \ldots, s_{n}\right\} \stackrel{\$}{\leftarrow} \mathbb{S}$ denote random sampling, but with some underlying probability distribution on $\mathbb{S}$. $\left(y_{1}, \ldots, y_{n}\right) \stackrel{\$}{\leftarrow} \mathcal{A}\left(x_{1}, \ldots, x_{m}\right)$ denotes a probabilistic algorithm $\mathcal{A}$ which takes as input $\left(x_{1}, \ldots, x_{m}\right)$ to produce output $\left(y_{1}, \ldots, y_{n}\right)$. Sometimes the internal coins $\rho$ of this algorithm is given explicitly as an input. This is distinguished from the normal input using a semi-colon, e.g., $y \leftarrow \mathcal{A}(x ; \rho)$.

Next, we introduce some notations pertaining to random oracles. The symbol $<$ is used to order the random oracle calls; e.g., $\mathrm{H}(x)<\mathrm{G}(y)$ indicates that the random oracle call $\mathrm{H}(x)$ precedes the random oracle call $\mathrm{G}(y)$. More generally, $\mathrm{H}<\mathrm{G}$ indicates that the target $\mathrm{H}$ oracle call precedes the target G-oracle call. The convention applies to hash functions as well. The symbol, on the other hand, $\prec$ is used to indicate random oracle dependence; e.g. $\mathrm{H} \prec \mathrm{G}$ indicates that the random oracle $\mathrm{G}$ is dependent on the random oracle $\mathrm{H}$. In the discussion involving the forking algorithms, $\mathrm{Q}_{j}^{i}$ denotes the $j^{\text {th }}$ random oracle query in round $\mathrm{i}$ of simulation.

Organisation of the paper. We take a closer look at the MF Algorithm in $\S 2$. In $\S \mathbf{3}$, we give an improved analysis of the MF Algorithm, while in $\S 4$, we apply the improvements to some of the existing schemes. Finally, we end with the concluding remarks in $\S \mathbf{5}$. As for the appendix, we begin with the GF Algorithm and the original MF Algorithm in Appendix A and B respectively. We give the deferred analyses of MF Algorithm with $\mathrm{O}_{\mathrm{I}}$ and $\mathrm{O}_{\mathrm{D}}$ (separately) in Appendix C. The construction of the schemes referred in the paper is given in Appendix D. We conclude with a section dedicated to the detailed security argument of the (modified) GG-IBS.

\section{Multiple-Forking: A Closer Look}

We begin with a critical look at the MF Algorithm and the associated lemma. A slightly revised version of the algorithm is given below (the original algorithm of [BPW12] is reproduced in Appendix B).

The Multiple-Forking Algorithm. Fix $q \in \mathbb{Z}^{+}$and a set $\mathbb{S}$ such that $|\mathbb{S}| \geq 2$. Let $\mathcal{Y}$ be a randomised algorithm that on input a string $x$ and elements $s_{1}, \ldots, s_{q} \in \mathbb{S}$ returns a triple 
$(I, J, \sigma)$ consisting of two integers $0 \leq J<I \leq q$ and a string $\sigma$. Let $n \geq 1$ be an odd integer. The MF Algorithm $\mathcal{M}_{\mathcal{Y}, n}$ associated to $\mathcal{Y}$ and $n$ is defined in Algorithm 1.

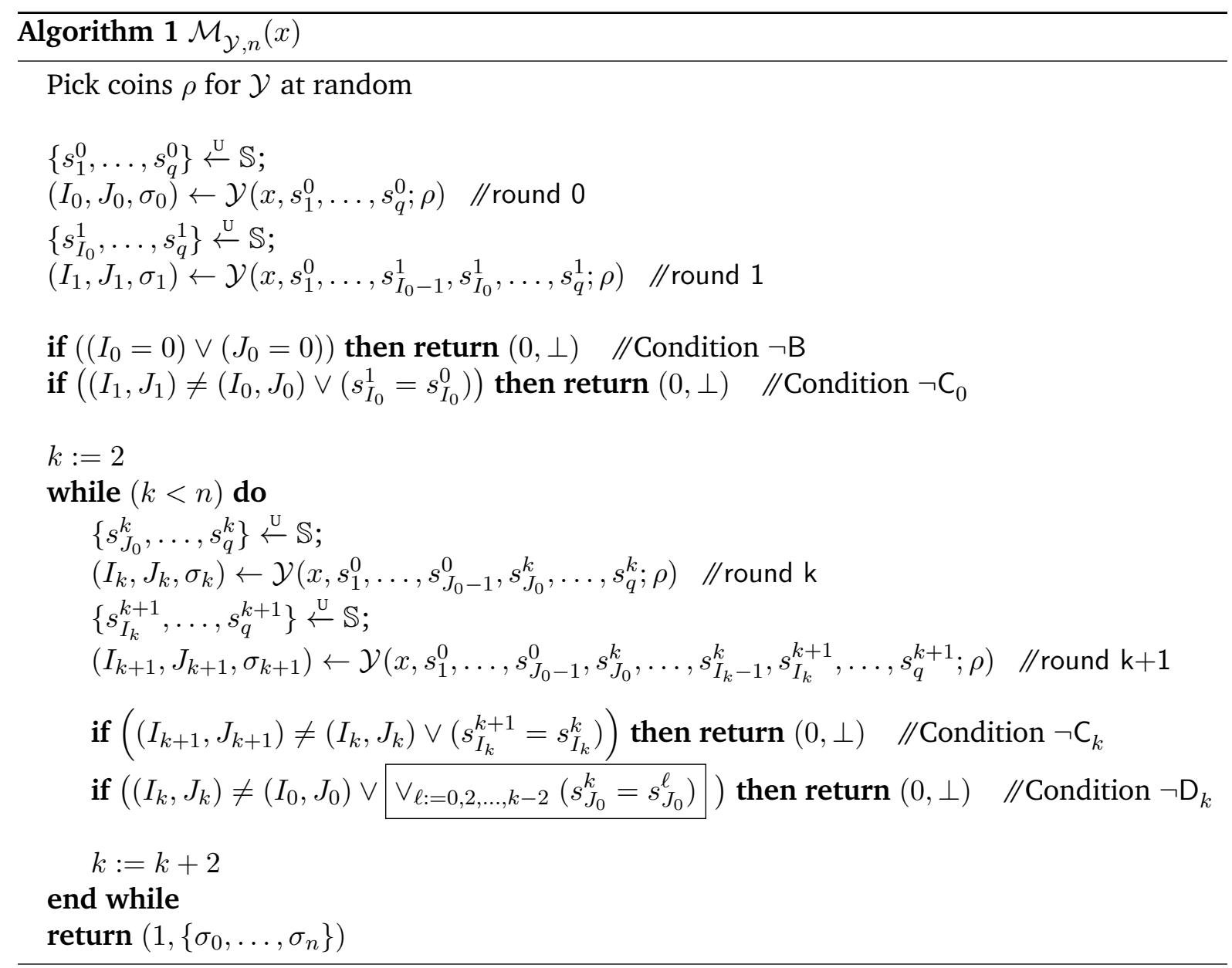

Note that we have introduced some conceptual changes in the MF Algorithm of [BPW12]. Before delving into the structure of the algorithm and ways to improve upon the bound on its probability of success, we briefly comment on a subtle (but minor in effect) logical flaw that we have fixed in the original version of [BPW12].

Remark 1. For the convenience of the readers we have boxed the modification in Algorithm 1. The original algorithm checked for $\left(s_{J_{0}}^{k}=s_{J_{0}}^{k-1}\right)$. However, this is not sufficient for some of the reduction algorithms that use the original $\mathcal{M}_{\mathcal{Y}, n}$. For example, consider the application of $\mathcal{M}_{\mathcal{Y}, 5}$ in the security argument of the [CMW12] protocol. At the end of the simulation, the MF Algorithm outputs six "cheating" transcripts $\left\{\left(v_{1}^{i}, c_{1}^{i}, s_{1}^{i}\right),\left(v_{1}^{i}, c_{2}^{i}, s_{2}^{i}\right)\right\}$, for $i:=1,2,3$, and the reduction, subsequently, finds the solution to the DLP by computing

$$
\left(\frac{z_{1} w_{1}-z_{2} w_{2}}{z_{1}-z_{2}}-\frac{z_{1} w_{1}-z_{3} w_{3}}{z_{1}-z_{3}}\right) /\left(\frac{w_{1}-w_{3}}{z_{1}-z_{3}}-\frac{w_{1}-w_{2}}{z_{1}-z_{2}}\right) \quad \bmod p
$$

where $w_{i}=\left(s_{1}^{i}-s_{2}^{i}\right) /\left(c_{2}^{i}-c_{1}^{i}\right)$ and where $z_{1}:=s_{J_{0}}^{0}, z_{2}:=s_{J_{0}}^{2}$ and $z_{3}:=s_{J_{0}}^{4}$ in the original $\mathcal{M}_{\mathcal{Y}, 5}$. Thus, for a correct solution of the discrete-log problem (DLP), the reduction requires to compute $\left(z_{1}-z_{2}\right)^{-1}$ and $\left(z_{1}-z_{3}\right)^{-1}$. [CMW12] asserts "[a]ccording to the probing strategy $z_{1}, z_{2}, z_{3}$ are all distinct". However, as per the original proposition, $\mathcal{M}_{\mathcal{Y}, 5}$ of [BPW12] will only ensure that $z_{2} \neq z_{1}$ and $z_{3} \neq z_{2}$ but not necessarily $z_{1} \neq z_{3}$. Hence, the probing strategy does not guarantee that all the $z_{i} \mathrm{~S}$ are distinct and the reduction may fail even though the MF Algorithm returns success. Clearly, the fault lies in the condition $\neg \mathrm{D}_{k}$ within the while loop of original 
MF Algorithm, which checks for equality only with the round just preceding it and comes into the picture for $\mathcal{M}_{\mathcal{Y}, n}$ for $n \geq 5$. A simple fix is to introduce pairwise check for equality, i.e., by changing the proposition from $\left(s_{J_{0}}^{k}=s_{J_{0}}^{k-1}\right)$ to $\left(\vee_{l:=0,2, \ldots, k-2} s_{J_{0}}^{k}=s_{J_{0}}^{\ell}\right)$. The change has a (small) bearing on the security bound given in the original MF Lemma due to an increase in the number of (inequality) checks from $n$ to $(n+1)(n+3) / 8$. This is captured in the revised version given below.

Lemma 1 (Revised Multiple-Forking Lemma). Let $\mathcal{G}_{I}$ be a randomised algorithm that takes no input and returns a string. Let

$$
\begin{gathered}
m f r k:=\operatorname{Pr}\left[(b=1) \mid x \stackrel{\&}{\leftarrow} \mathcal{G}_{I} ;\left(b,\left\{\sigma_{0}, \ldots, \sigma_{n}\right\}\right) \stackrel{\$}{\leftarrow} \mathcal{M}_{\mathcal{Y}, n}(x)\right] \text { and } \\
\text { acc }:=\operatorname{Pr}\left[(I \geq 1) \wedge(J \geq 1) \mid x \stackrel{\&}{\leftarrow} \mathcal{G}_{I} ;\left\{s_{1}, \ldots, s_{q}\right\} \stackrel{\cup}{\leftarrow} \mathbb{S} ;(I, J, \sigma) \stackrel{\$}{\leftarrow} \mathcal{Y}\left(x, s_{1}, \ldots, s_{q}\right)\right]
\end{gathered}
$$

then

$$
m f r k \geq a c c \cdot\left(\frac{a c c^{n}}{q^{2 n}}-\frac{(n+1)(n+3)}{8|\mathbb{S}|}\right) .
$$

\subsection{Tightness: An Intuitive Picture}

Each run of $\mathcal{M}_{\mathcal{Y}, n}$ consists of $n+1$ runs of the corresponding wrapper $\mathcal{Y}$ (called round 0 to round $\mathrm{n}$ ), for some odd $n$. Informally speaking, $\mathcal{M}_{\mathcal{Y}, n}$ is successful provided $\mathcal{Y}$ is successful in each of the $n+1$ rounds and some additional conditions are satisfied. We call these set of conditions $\mathbb{A}_{0}:=\left\{\mathrm{B}, \mathrm{C}_{0}, \ldots, \mathrm{C}_{n-1}, \mathrm{D}_{2}, \ldots, \mathrm{D}_{n-1}\right\}$ where

$$
\begin{aligned}
\mathrm{B} & :\left(I_{0} \geq 1\right) \wedge\left(J_{0} \geq 1\right) \\
\mathrm{C}_{k} & :\left(I_{k+1}, J_{k+1}\right)=\left(I_{k}, J_{k}\right) \wedge\left(s_{I_{k}}^{k+1} \neq s_{I_{k}}^{k}\right)(\text { for } k=0,2, \ldots, n-1) \\
\mathrm{D}_{k} & :\left(I_{k}, J_{k}\right)=\left(I_{0}, J_{0}\right) \wedge\left(\wedge_{l:=0,2, \ldots, k-2} s_{J_{0}}^{k} \neq s_{J_{0}}^{\ell}\right)(\text { for } k=2,4, \ldots, n-1)
\end{aligned}
$$

Let $E$ be the event that all the conditions in $\mathbb{A}_{0}$ are satisfied, i.e.,

$$
E: B \wedge\left(C_{0} \wedge C_{2} \wedge \cdots \wedge C_{n-1}\right) \wedge\left(D_{2} \wedge D_{4} \wedge \cdots \wedge D_{n-1}\right)
$$

What the MF Lemma then gives us is, essentially, a lower bound of the probability of this event.

The logical wrapper $\mathcal{Z}$. A simple but crucial observation at this point is that the wrapper algorithm $\mathcal{Y}$ is always invoked in pairs (that's the reason for $n$ being odd in the description of $\mathcal{M}_{\mathcal{Y}, n}$ ). Note that, the conditions $\mathrm{C}_{k}$ and $\mathrm{D}_{k}$ above also pertain to a pair of invocations. This brings us to the conceptual change introduced in the revised version given in Algorithm 1. Two consecutive invocations of $\mathcal{Y}$ (i.e., round $\mathrm{k}$ and round $\mathrm{k}+1$, for even $k \geq 0$ ) have been clubbed together so that it can be visualised (see Figure 2) as the invocation of a single logical unit $\mathcal{Z}$ such that $\left(\left(I_{k}, J_{k}, \sigma_{k}\right),\left(I_{k+1}, J_{k+1}, \sigma_{k+1}\right)\right) \leftarrow \mathcal{Z}\left(x, \mathrm{~S}^{k}, \mathrm{~S}^{k+1} ; \rho\right)$ (for even $k$ ). Here, $\mathrm{S}^{k}$ and $\mathrm{S}^{k+1}$ denote the external random coins for the rounds $k$ and $k+1$ of $\mathcal{Y}$, i.e.,

$$
\mathrm{S}^{k}:=\left(s_{1}^{0}, \ldots, s_{J_{0}-1}^{0}, s_{J_{0}}^{k}, \ldots, s_{q}^{k}\right) \text { and } \mathrm{S}^{k+1}:=\left(s_{1}^{0}, \ldots, s_{J_{0}-1}^{0}, s_{J_{0}}^{k}, \ldots, s_{I_{k}-1}^{k}, s_{I_{k}}^{k+1}, \ldots, s_{q}^{k+1}\right) \text {. }
$$

Accordingly, the MF Algorithm constitutes of $m=(n+1) / 2$ rounds of invocation of $\mathcal{Z}$. Intuitively, a single invocation of $\mathcal{Z}$ is similar to the GF Algorithm [BN06] as the objective of both is to launch the "elementary" oracle replay attack. The notion of wrapper $\mathcal{Z}$, along with the necessary restructuring of the MF Algorithm-especially, the conditions-provide us the right handle for an improved analysis of its probability of success ( $m f r k$ ). 


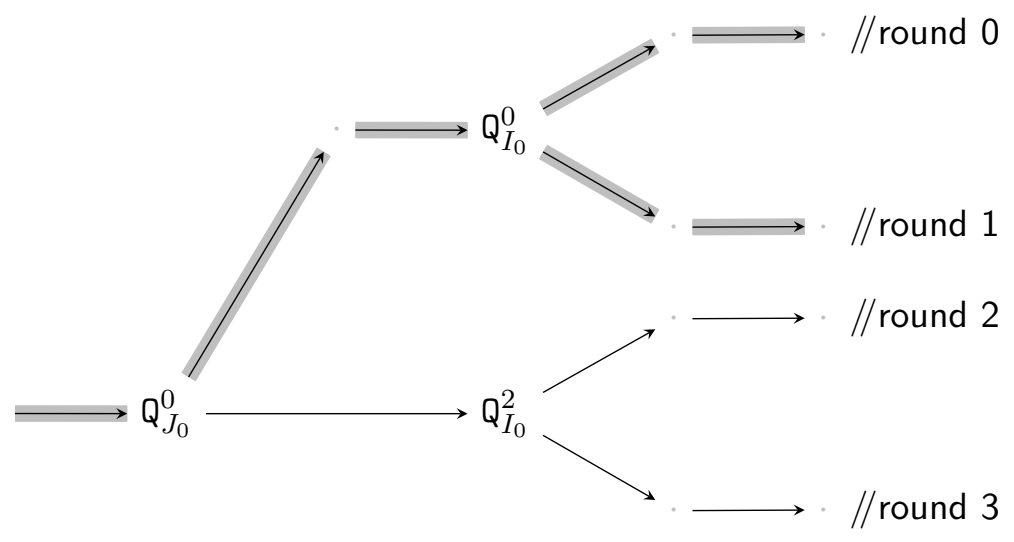

Figure 2: The logical wrapper $\mathcal{Z}$. One such logical wrapper (for round 0 and round 1 ) has been highlighted.

Among the set of conditions $\mathbb{A}_{0}$, $B$ is relatively easy to deal with as it is checked only at the beginning and contributes a factor of acc in the final expression for $m f r k$. So let's look at the effect of the other (more involved) conditions. Consider the event

$$
\mathrm{C}_{k}:\left(I_{k+1}, J_{k+1}\right)=\left(I_{k}, J_{k}\right) \wedge\left(s_{I_{0}}^{k+1} \neq s_{I_{0}}^{k}\right) .
$$

Clearly, the check for equality $\left(I_{k+1}, J_{k+1}\right)=\left(I_{k}, J_{k}\right)$ is predominant in the final expression for $m f r k$ (the equality holds only with a probability of $\left.1 / q^{2}\right)$. On the other hand, $\left(s_{I_{0}}^{k+1} \neq s_{I_{0}}^{k}\right)$ is almost always true ${ }^{7}$. It's a similar case for the event $\mathrm{D}_{k}:\left(I_{k}, J_{k}\right)=\left(I_{0}, J_{0}\right)$ holds only with a probability of $1 / q^{2}$. Hence, the probability of the events $C$ and $\mathrm{D}$ is dominated respectively by

$$
\wedge_{k:=0,2, \ldots, n-1}\left(I_{k+1}, J_{k+1}\right)=\left(I_{k}, J_{k}\right) \text { and } \wedge_{k:=2,4, \ldots, n-1}\left(I_{k}, J_{k}\right)=\left(I_{0}, J_{0}\right) .
$$

Consequently, the degradation for the MF Algorithm stems, predominantly, from what we term as the "core" event ${ }^{8}$

$$
\mathrm{F}_{0}:\left(I_{n}, J_{n}\right)=\left(I_{n-1}, J_{n-1}\right)=\cdots=\left(I_{0}, J_{0}\right)
$$

formed by combining the two expressions in (4). Each of the $n$ checks for equality in the condition contributes (roughly speaking) a factor of $\mathrm{O}\left(q^{2}\right)$, resulting in an overall degradation of $\mathrm{O}\left(q^{2 n}\right)$. That's the intuitive reason of the (loose) lower bound one gets for $m f r k$. Naturally, any reduction employing $\mathcal{M}_{\mathcal{Y}, n}$ also loses tightness by a factor of $\mathrm{O}\left(q^{2 n}\right)$.

\subsection{Road-map to a Better Analysis}

By now it should be evident that in order to achieve a better bound for $m f r k$, one needs to revisit the conditions associated with the number of checks involved in the success event $F_{0}$. For a better understanding of the exact role of these conditions, we revisit the concrete protocols and their security argument that employ the MF Lemma [BPW12, GG09, CMW12]. Based on a careful analysis of these protocols and their security arguments, we formulate the following two observations-called, respectively, the independence and the dependence conditions.

Observation 1 (Independence condition, $\mathrm{O}_{\mathrm{I}}$ ). It is not necessary for the I indices across the logical wrapper $\mathcal{Z}$ to be the same, i.e., $I_{k}$ need not be equal to $I_{k-2}, I_{k-4}, \ldots, I_{0}$ for $k=2,4, \ldots, n-1$.

\footnotetext{
${ }^{7}$ It holds with a probability of $(1-(1 /|\mathbb{S}|))$ and for any reasonable security level, $|\mathbb{S}| \gg 1$.

${ }^{8}$ Note that the event $F_{0}$ corresponds to the first term of (C.3) in the analysis of the MF Algorithm given in [BPW12].
} 
Observation 2 (Dependence condition, $\mathrm{O}_{\mathrm{D}}$ ). It is possible to design protocols, in particular, define hash functions used in the protocol, such that, for the $k^{\text {th }}$ invocation of the logical wrapper $\mathcal{Z}$, $\left(I_{k+1}=I_{k}\right)$ implies (with very high probability) that $\left(J_{k+1}=J_{k}\right){ }^{9}$

Remark 2. The concrete motivation for the above two observations will become clear when we revisit the actual security argument of the existing schemes in $\S 4 . \mathrm{O}_{\mathrm{I}}$ is based on a precise analysis of what is actually required from the process of (multiple) forking in the security argument of [BPW12, GG09, CMW12]. In particular, all known applications of MF Algorithm satisfies $\mathrm{O}_{\mathrm{I}}$. $\mathrm{O}_{\mathrm{D}}$ finds its root in the more concrete notion of hash function dependence. Intuitively, if a protocol uses two hash functions $\mathrm{H}_{1}$ and $\mathrm{H}_{2}$ in such a way that the input to $\mathrm{H}_{2}$ is a function of the output of $\mathrm{H}_{1}$, then we say that the $\mathrm{H}_{2}$-call is dependent on the $\mathrm{H}_{1}$-call. The $\mathrm{BPW}$ proxy signature scheme of [BPW12] is one example where such dependence exists (see $\S 4.1$ for further details). As in the case of $\mathrm{O}_{I}$, we'll show that either $\mathrm{O}_{\mathrm{D}}$ is naturally satisfied [CMW12] or one can easily modify existing construction [GG09] to suit the condition (see $\S 4.4$ and $\S 4.3 .1$ resp.). The abstraction is required in the context of MF Algorithm due to the absence of the notion of hash function (or random oracles, which model the hash functions in the actual security argument). A formal definition of the notion of index dependence, keeping in mind the observation $O_{D}$, is given below.

Definition 1 (Index Dependence). Let $\left((I, J),\left(I^{\prime}, J^{\prime}\right)\right)$ be the two pair of indices that are part of the output of the wrapper $\mathcal{Z}$ (associated with two rounds of $\mathcal{Y}$ ). The index $J$ is said to be $\eta$-dependent on the index $I$ (denoted by $J \prec I$ ) if $\operatorname{Pr}\left[J^{\prime} \neq J \mid I^{\prime}=I\right] \leq \eta$.

In other words, $\left(I^{\prime}=I\right) \Longrightarrow\left(J^{\prime}=J\right)$ with probability at least $(1-\eta)$. A little more specifically, $J$ is said to be fully-dependent on $I$ if $\eta=0$, i.e. $\left(I^{\prime}=I\right) \Longrightarrow\left(J^{\prime}=J\right)$. But for most applications, it suffices that $J$ be $\eta$-dependent on $I$ for some $\eta$ which is negligible ${ }^{10}$ in the security parameter $\kappa$.

Consequences. Let's look at the consequences of the observations one by one. Based on $\mathrm{O}_{\mathrm{I}}$, the condition in (4) can be relaxed to: $\wedge_{k:=0,2, \ldots, n-1}\left(I_{k+1}, J_{k+1}\right)=\left(I_{k}, J_{k}\right)$ and $\wedge_{k:=2,4, \ldots, n-1}\left(J_{k}=\right.$ $J_{0}$ ), and, by implication, the "core" event in (5) is relaxed to

$$
\mathrm{F}_{1}: \wedge_{k=0,2, \ldots, n-1}\left(\left(I_{k+1}, J_{k+1}\right)=\left(I_{k}, J_{k}\right)\right) \wedge\left(J_{n-1}=J_{n-3}=\cdots=J_{0}\right) .
$$

Hence, the number of overall checks (in the "core" event) is reduced from $2 n$ to $(2 \cdot(n+1) / 2+$ $(n-1) / 2)=(3 n+1) / 2$ and the complexity of launching the nested oracle replay attack is brought down to $\mathrm{O}\left(q^{(3 n+1) / 2}\right)$. A similar analysis shows that based on only $\mathrm{O}_{\mathrm{D}}$ (and, assuming $\eta$ to be negligible in $\kappa$ ), the "core" event in (5) relaxes to

$$
\mathrm{F}_{2}:\left(I_{n}=I_{n-1}=\cdots=I_{1}=I_{0}\right) \wedge\left(J_{n-1}=J_{n-3}=\cdots=J_{0}\right)
$$

In this case the number of overall checks is reduced from $2 n$ to $(3 n-1) / 2$ and the complexity to $\mathrm{O}\left(q^{(3 n-1) / 2}\right)$. The interesting point is that $\mathrm{O}_{\mathrm{I}}$ and $\mathrm{O}_{\mathrm{D}}$ can be employed in conjunction. This leads to the "core" event being further simplified to

$$
\mathrm{F}_{3}:\left(I_{n}=I_{n-1}\right) \wedge \cdots \wedge\left(I_{1}=I_{0}\right) \wedge\left(J_{n-1}=J_{n-3}=\cdots=J_{0}\right) .
$$

Observe that the number of checks is now reduced to $n$ and the complexity to $\mathrm{O}\left(q^{n}\right)$. Hence, the complexity of launching the nested oracle replay attack is reduced from $\mathrm{O}\left(q^{2 n}\right)$ to $\mathrm{O}\left(q^{n}\right)$, but, without losing the modularity of the MF Algorithm.

\footnotetext{
${ }^{9}$ Assume, without loss of generality, that among the two indices, $J$ always precedes $I$ in a particular run of the wrapper.

${ }^{10}$ A function $\mathrm{f}: \mathbb{R} \mapsto \mathbb{R}$ is negligible if for any $n>0$, we have $|\mathrm{f}(x)|<1 / k^{n}$ for sufficiently large $x$ [BF01].
} 


\subsection{A General Multiple-Forking Algorithm}

As we just observed, depending on whether (or not) the observations $\mathrm{O}_{I}$ and $\mathrm{O}_{D}$ are taken into account, we end up with four different sets of "core" conditions $F_{0}$ through $F_{3}$. The "non-core" conditions, though, remain the same for all the four cases. The resulting set of "full" conditions, $\mathbb{A}_{0}$ through $\mathbb{A}_{3}$, (along with the associated degradation) are given in Table 2. In order to capture this extra level of abstraction, we describe a general framework of the MF Algorithm that has, in addition to the algorithm $\mathcal{Y}$, an associated (ordered) set of conditions $\mathbb{A}$. Note that we have assumed full-dependence in formulating the table.

\begin{tabular}{|c|c|c|}
\hline MF & Set of Conditions & Degradation \\
\hline Original & $\mathbb{A}_{0}= \begin{cases}\mathrm{B}: & \left(I_{0} \geq 1\right) \wedge\left(J_{0} \geq 1\right) \\
\mathrm{C}_{k}: & \left(I_{k+1}, J_{k+1}\right)=\left(I_{k}, J_{k}\right) \wedge\left(s_{I_{k}}^{k+1} \neq s_{I_{k}}^{k}\right) \\
\mathrm{D}_{k}: & \left(I_{k}, J_{k}\right)=\left(I_{0}, J_{0}\right) \wedge\left(\wedge_{\ell:=0,2, \ldots, k-2} s_{J_{0}}^{k} \neq s_{J_{0}}^{\ell}\right)\end{cases}$ & $\mathrm{O}\left(q^{2 n}\right)$ \\
\hline with $\mathrm{O}_{\mathrm{I}}$ & $\mathbb{A}_{1}= \begin{cases}\mathrm{B}: & \left(I_{0} \geq 1\right) \wedge\left(J_{0} \geq 1\right) \\
\mathrm{C}_{k}: & \left(I_{k+1}, J_{k+1}\right)=\left(I_{k}, J_{k}\right) \wedge\left(s_{I_{k}}^{k+1} \neq s_{I_{k}}^{k}\right) \\
\mathrm{D}_{k}: & \left(J_{k}=J_{0}\right) \wedge\left(I_{k} \geq 1\right) \wedge\left(\wedge_{\ell:=0,2, \ldots, k-2} s_{J_{0}}^{k} \neq s_{J_{0}}^{\ell}\right)\end{cases}$ & $\mathrm{O}\left(q^{(3 n+1) / 2}\right)$ \\
\hline with $\mathrm{O}_{\mathrm{D}}$ & $\mathbb{A}_{2}=\left\{\begin{array}{lll}\mathrm{B}: & \left(1 \leq J_{0}<I_{0} \leq q\right) \\
\mathrm{C}_{k}: & \left(I_{k+1}=I_{k}\right) \wedge\left(s_{I_{k}}^{k+1} \neq s_{I_{k}}^{k}\right) \\
\mathrm{D}_{k}: & \left(I_{k}, J_{k}\right)=\left(I_{0}, J_{0}\right) \wedge\left(\wedge_{\ell:=0,2, \ldots, k-2} s_{J_{0}}^{k} \neq s_{J_{0}}^{\ell}\right)\end{array}\right.$ & $\mathrm{O}\left(q^{(3 n-1) / 2}\right)$ \\
\hline with $\mathrm{O}_{\{\mathrm{I}, \mathrm{D}\}}$ & $\mathbb{A}_{3}=\left\{\begin{array}{lll}\mathrm{B}: & \left(1 \leq J_{0}<I_{0} \leq q\right) \\
\mathrm{C}_{k}: & \left(I_{k+1}=I_{k}\right) \wedge\left(s_{I_{k}}^{k+1} \neq s_{I_{k}}^{k}\right) \\
\mathrm{D}_{k}: & \left(J_{k}=J_{0}\right) \wedge\left(J_{k}<I_{k} \leq q\right) \wedge\left(\wedge_{\ell:=0,2, \ldots, k-2} s_{J_{0}}^{k} \neq s_{J_{0}}^{\ell}\right)\end{array}\right.$ & $\mathrm{O}\left(q^{n}\right)$ \\
\hline
\end{tabular}

Table 2: The set of conditions is $\mathbb{A}:=\{B, C, D\}$ where $C$ denotes $C_{0}, C_{2}, \ldots, C_{n-1}$ and $D$ denotes $\mathrm{D}_{2}, \mathrm{D}_{4}, \ldots, \mathrm{D}_{n-1}$. Also, note that we have ignored the (common) $\epsilon^{n}$ factor in the degradation and assumed full-dependence.

The General Multiple-Forking Algorithm Fix $q \in \mathbb{Z}^{+}$and a set $\mathbb{S}$ such that $|\mathbb{S}| \geq 2$. Let $\mathcal{Y}$ be a randomised algorithm that on input a string $x$ and elements $s_{1}, \ldots, s_{q} \in \mathbb{S}$ returns a triple $(I, J, \sigma)$ consisting of two integers $0 \leq J<I \leq q$ and a string $\sigma$. Let $n \geq 1$ be an odd integer. In addition, let $\mathbb{A}$ denote the set of conditions, and be of the form $\{B, C, D\}$ with $\mathrm{C}:=\mathrm{C}_{0}, \mathrm{C}_{2}, \ldots, \mathrm{C}_{n-1}$ and $\mathrm{D}:=\mathrm{D}_{2}, \mathrm{D}_{4}, \ldots, \mathrm{D}_{n-1}$. The General MF Algorithm $\mathcal{N}_{\mathbb{A}, \mathcal{Y}, n}$ associated to $\mathbb{A}, \mathcal{Y}$ and $n$ is defined as Algorithm 2 below. 


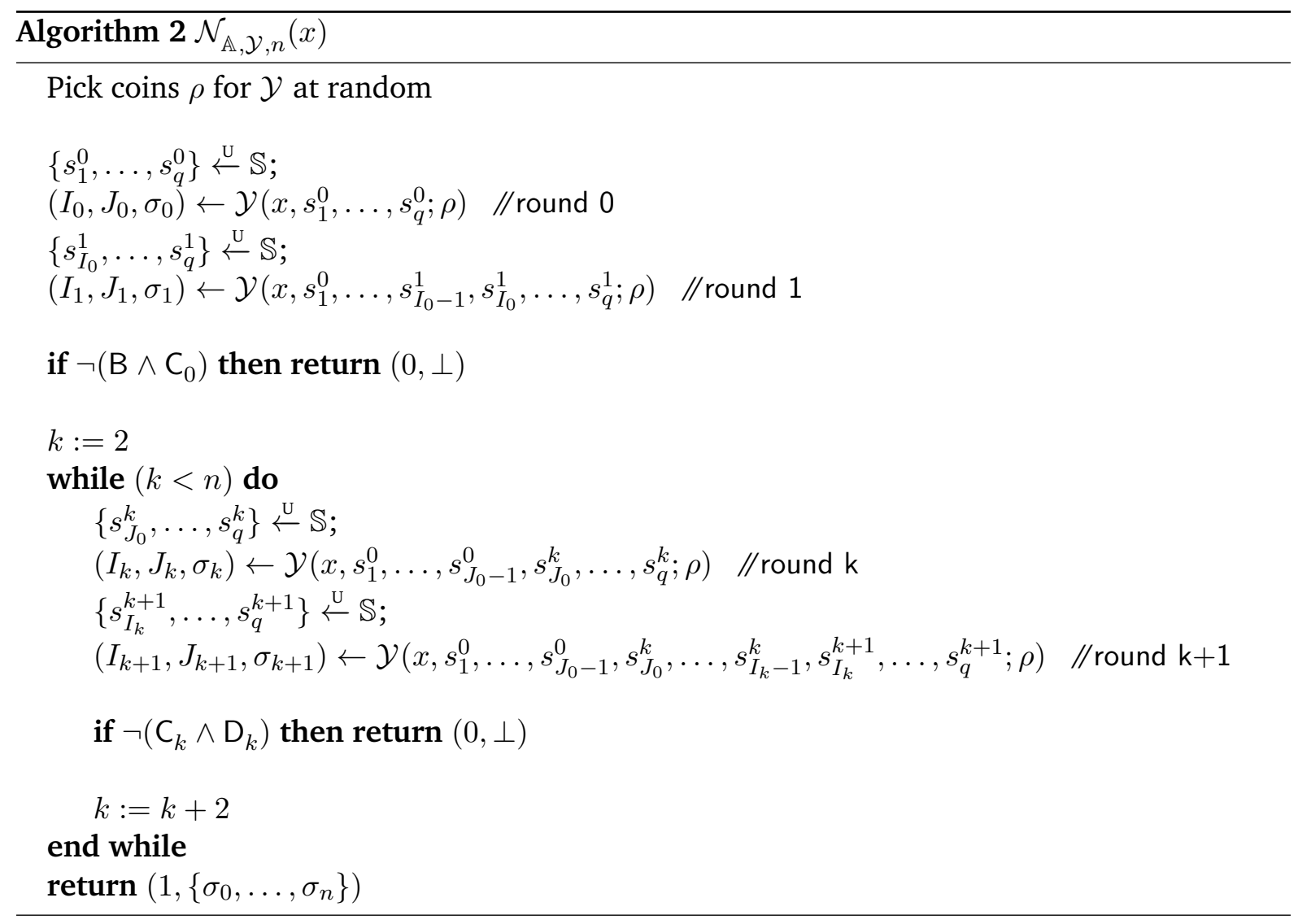

Remark 3 (On usage). We use the set of conditions $\mathbb{A}_{2}$ and $\mathbb{A}_{3}$ only in the case of $i$ ) fulldependence; or ii) $\eta$-dependence with negligible $\eta$. For $\eta$-dependence, in general, we use the set of conditions $\mathbb{A}_{0}$ and $\mathbb{A}_{1}$ respectively (e.g., see Lemma 2 and its proof).

\section{Harnessing (In)Dependence}

We have seen in the previous section that the most effective way of launching the nested replay attack is by exploiting both $\mathrm{O}_{\mathrm{I}}$ and $\mathrm{O}_{\mathrm{D}}$. As expected, the analysis of this case turns out to be the most involved and in a sense subsumes the analysis of the other two set of conditions $\left(\mathbb{A}_{1}\right.$ and $\mathbb{A}_{2}$ ). Hence in this section we focus on analysing $\mathcal{N}_{\mathbb{A}_{3}, \mathcal{Y}, n}$ with $\eta$-dependence. The analysis of $\mathcal{N}_{\mathbb{A}_{1}, \mathcal{Y}, n}$ and $\mathcal{N}_{\mathbb{A}_{2}, \mathcal{Y}, n}$ with $\eta$-dependence is deferred to Appendix $\mathbf{C}$ (see Lemma 9 and Lemma 10).

\subsection{Multiple-Forking with Index (In)Dependence}

The probability of success of the MF Algorithm with both $\mathrm{O}_{I}$ and $\mathrm{O}_{\mathrm{D}}$ is bounded by Lemma 2 given below. The details of some of the steps in the probability analysis is given in Appendix C.1.

Lemma 2 (Multiple-Forking Lemma with Index (In)Dependence). Let $\mathcal{G}_{I}$ be a randomised algorithm that takes no input and returns a string. Let

$$
\begin{gathered}
m f r k_{3}:=\operatorname{Pr}\left[(b=1) \mid x \stackrel{\$}{\leftarrow} \mathcal{G}_{I} ;\left(b,\left\{\sigma_{0}, \ldots, \sigma_{n}\right\}\right) \stackrel{\$}{\leftarrow} \mathcal{N}_{\mathbb{A}_{1}, \mathcal{Y}, n}(x)\right] \text { and } \\
a c c:=\operatorname{Pr}\left[(1 \leq J<I \leq q) \mid x \stackrel{\$}{\leftarrow} \mathcal{G}_{I} ;\left\{s_{1}, \ldots, s_{q}\right\} \stackrel{\cup}{\leftarrow} \mathbb{S} ;(I, J, \sigma) \stackrel{\$}{\leftarrow} \mathcal{Y}\left(x, s_{1}, \ldots, s_{q}\right)\right] .
\end{gathered}
$$


On the assumption that $J$ is $\eta$-dependent on $I$,

$$
m f r k_{3} \geq f r k\left(\frac{f r k^{(n-1) / 2}}{q^{(n-1) / 2}}-\frac{(n-1)(n+1)}{8|\mathbb{S}|}\right) \text { where frk } \geq \operatorname{acc}\left(\frac{\operatorname{acc}}{q}(1-\eta)-\frac{1}{|\mathbb{S}|}\right)
$$

The main hurdle in proving the bounds lies in exploiting the leverage offered by $\mathrm{O}_{\mathrm{I}}$ and $\mathrm{O}_{\mathrm{D}}$, in the probability analysis. As it turns out, $\mathrm{O}_{\mathrm{D}}$ is much harder to integrate. We have to appeal to the underlying sets (i.e. the source of randomness) of the GMF Algorithm. On the other hand, the analysis of $\mathrm{O}_{\mathrm{I}}$ is in some sense similar to the analysis of the original MF Algorithm in [BPW12]. Naturally, the simultaneous analysis of $\mathrm{O}_{\mathrm{I}}$ and $\mathrm{O}_{\mathrm{D}}$ is a "hybrid" of the above two. The subtle change in the accepting condition to $(1 \leq J<I \leq q)$ from $(I \geq 1) \wedge(J \geq 1)$ in Lemma 1 is also crucial in establishing the bounds (see Claim 3 below). The following two inequalities are also used.

Lemma 3 (Jensen's inequality). Let $f$ be a convex function and $X$ be a real-valued random variable. Then

$$
\operatorname{Ex}[f(X)] \geq f(\operatorname{Ex}[X]) .
$$

Lemma 4 (Hölder's inequality ${ }^{11}$ ). Let $q \in \mathbb{Z}^{+}, 1 \leq n<\infty$ and $x_{1}, \ldots, x_{q} \geq 0$ be real numbers. Then

$$
\sum_{k:=1}^{q} x_{k}^{n} \geq \frac{1}{q^{n-1}}\left(\sum_{k:=1}^{q} x_{k}\right)^{n} \text {. }
$$

Conventions. Before moving on to the proof, we fix some conventions with regard to the coins involved in the GMF Algorithm. The internal coins for the algorithm $\mathcal{Y}$ is denoted by $\rho$. We assume that $\rho$ is drawn from a set $\mathbb{R}$ (not to be confused with the set of real numbers). On the other hand, the external randomness for $\mathcal{Y}$ is denoted by $\mathrm{S}:=\left\{s_{1}, \ldots, s_{q}\right\}$ (with the round indicated in the superscript, e.g. $\mathrm{S}^{0}$ ). For convenience, we use the convention in Table 3 to split up S. Hence, $\left\{\mathrm{S}_{(i-)}, \mathrm{S}_{(i+)}\right\}$ indicates $\mathrm{S}$ split up into two at the index $i$, whereas $\left\{\mathrm{S}_{(j-)}, \mathrm{S}_{(j i)}, \mathrm{S}_{(i+)}\right\}$

\begin{tabular}{ccc} 
Symbol & Denotes & Domain \\
\hline $\mathbf{S}_{(i-)}$ & $s_{1}, \ldots, s_{i-1}$ & $|\mathbb{S}|^{i-1}$ \\
$\mathbf{S}_{(j i)}$ & $s_{j}, \ldots, s_{i-1}$ & $|\mathbb{S}|^{i-j}$ \\
$\mathbf{S}_{(i+)}$ & $s_{i}, \ldots, s_{q}$ & $|\mathbb{S}|^{q-i+1}$ \\
\hline
\end{tabular}

Table 3: Shorthand for external randomness.

indicates $\mathrm{S}$ split into three, at indices $j$ and $i$ respectively. Finally, $\mathbb{T}_{(2)}$ denotes the set from which coins (both internal and external) are drawn for $\mathcal{Z}$ and $\mathbb{T}_{(n)}$ denotes the set of coins for the GMF Algorithm ${ }^{12}$.

\footnotetext{
${ }^{11}$ Although, the result is a corollary to a more general Hölder's inequality (see [Lemma C.3][BPW12]), another way to proving the bound is by viewing it an optimisation problem. Let $\mathrm{f}\left(x_{1}, \ldots, x_{q}\right):=\sum_{k:=1}^{q} x_{k}^{n}$ be the objective function under the set of constraints: i) $\sum_{k=1}^{q} x_{k}=x$; and ii) $\left(0 \leq x_{k} \leq 1\right)$ for $k \in\{1, \ldots, q\}$. Then $\mathrm{f}$ attains a minima of $x^{n} / q^{n-1}$ at the point $(x / q, \ldots, x / q)$, thus, establishing Lemma 4.

${ }^{12}$ With regard to the coins, it is apparent that the source of coins for a single invocation of $\mathcal{Y}$ is the set $\mathbb{R} \times \mathbb{S}^{q}$. As for two invocations of $\mathcal{Y}$ (single invocation of $\mathcal{Z}$ ), it can be worked out that the coins are drawn from the set

$$
\mathbb{T}_{(2)}:=\mathbb{R} \times \bigcup_{i=2}^{q} \mathbb{S}^{i-1} \times \mathbb{S}^{(q-i+1) * 2}
$$
}


Proof. For a fixed string $x$, let

$$
\begin{gathered}
m \operatorname{mrk}_{3}(x):=\operatorname{Pr}\left[(\mathrm{b}=1) \mid\left(\mathrm{b},\left\{\sigma_{0}, \ldots, \sigma_{n}\right\}\right) \stackrel{\$}{\leftarrow} \mathcal{N}_{\mathbb{A}_{1}, \mathcal{Y}, n}(x)\right] \text { and } \\
\operatorname{acc}(x):=\operatorname{Pr}\left[(1 \leq J<I \leq q) \mid\left\{s_{1}, \ldots, s_{q}\right\} \stackrel{\mathrm{U}}{\leftarrow} \mathbb{S} ;(I, J, \sigma) \stackrel{\$}{\leftarrow} \mathcal{Y}\left(x, s_{1}, \ldots, s_{q}\right)\right] .
\end{gathered}
$$

Let $\mathbb{A}_{1}:=\left\{B, C_{0}, C_{2}, \ldots, C_{n-1}, D_{2}, D_{4}, \ldots, D_{n-1}\right\}$. For ease of notation, we further break the event $\mathrm{C}_{k}$ (resp. $\mathrm{D}_{k}$ ) into two subevents $\mathrm{C}_{k, c}$ and $\mathrm{C}_{k, s}$ (resp. $\mathrm{D}_{k, c}$ and $\mathrm{D}_{k, s}$ ) as shown below.

$$
\begin{aligned}
\mathrm{C}_{k, c}:\left(I_{k+1}=I_{k}\right) & \mathrm{C}_{k, s}:\left(s_{I_{k}}^{k+1} \neq s_{I_{k}}^{k}\right) \\
\mathrm{D}_{k, c}:\left(J_{k}=J_{0}\right) \wedge\left(J_{k}<I_{k} \leq q\right) & \mathrm{D}_{k, s}:\left(\wedge_{\ell:=0,2, \ldots, k-2} s_{J_{0}}^{k} \neq s_{J_{0}}^{\ell}\right)
\end{aligned}
$$

The GMF Algorithm is successful in the event $E: B \wedge\left(C_{0} \wedge C_{2} \wedge \cdots \wedge C_{n-1}\right) \wedge\left(D_{2} \wedge D_{4} \wedge \cdots \wedge D_{n-1}\right)$. In other words, with the probabilities calculated over the coin tosses of the GMF Algorithm, it follows that $m f r k_{3}(x)=\operatorname{Pr}[E]$. The task of bounding this probability is accomplished through three claims (Claim 1 through Claim 3). The object at the centre of Claim $\mathbf{1}$ is the logical unit $\mathcal{Z}$. It turns out that with index dependence, the behaviour of $\mathcal{Z}$ is similar to the GF Algorithm [BN06]. The aim of Claim 1 is to bound the probability of success of $\mathcal{Z}$, denoted by frk $(x)$, in terms of $\operatorname{acc}(x)$. The bound on $f r k(x)$ is, then, used in Claim 2 and Claim $\mathbf{3}$ to bound $m f r k k_{3}(x)$.

We start with the analysis of a single invocation of $\mathcal{Z}$. Without loss of generality, let's consider the first two rounds of the GMF Algorithm.

Claim 1. $\operatorname{frk}(x) \geq \operatorname{acc}(x)\left(\frac{\operatorname{acc}(x)}{q}(1-\eta(x))-\frac{1}{|\mathbb{S}|}\right)$.

Argument. With the probability taken over the coin tosses over the two rounds (which we denote by $\mathbb{T}_{(2)}$ ), it follows that

$$
\begin{aligned}
\operatorname{frk}(x) & =\operatorname{Pr}\left[\mathrm{B} \wedge \mathrm{C}_{0}\right]=\operatorname{Pr}\left[\mathrm{B} \wedge \mathrm{C}_{0, c} \wedge \mathrm{C}_{0, s}\right] \quad \text { (using subevents given in (10)) } \\
& \geq \operatorname{Pr}\left[\left(J_{1}=J_{0}\right) \wedge\left(I_{1}=I_{0}\right) \wedge\left(1 \leq J_{0}<I_{0} \leq q\right)\right]-\operatorname{Pr}\left[\left(1 \leq J_{0}<I_{0} \leq q\right) \wedge\left(s_{I_{0}}^{0}=s_{I_{0}}^{1}\right)\right]
\end{aligned}
$$

Let's denote the two parts of the above expression by $f r k_{c}(x)$ and $f r k_{s}(x)$ respectively. The second part can be computed as follows:

$$
\operatorname{frk}_{s}(x)=\operatorname{Pr}\left[1 \leq J_{0}<I_{0} \leq q\right] \cdot \operatorname{Pr}\left[s_{I_{0}}^{0}=s_{I_{0}}^{1}\right]=\operatorname{acc}(x) /|\mathbb{S}| .
$$

The first part of (11), on the other hand, forms the "core" probability for the two rounds. In order to analyse it, we define a random variable which captures single invocation of the algorithm $\mathcal{Y}$. Let $Y_{i}: \mathbb{R} \times \mathbb{S}^{i-1} \mapsto[0,1]$, for each $i \in\{2, \ldots, q\}$, be defined by setting

$$
\left.Y_{i}\left(\rho, \mathrm{S}_{(i-)}\right)=\operatorname{Pr}[(I=i) \wedge(1 \leq J<i)) \mid \mathrm{S}_{(i+)} \stackrel{\amalg}{\leftarrow} \mathbb{S} ;(I, J, \sigma) \leftarrow \mathcal{Y}\left(x,\left\{\mathrm{~S}_{(i-)}, \mathrm{S}_{(i+)}\right\} ; \rho\right)\right] .
$$

By a simple extrapolation, the coins for the GMF Algorithm are drawn from

$$
\mathbb{T}_{(n)}:=\mathbb{R} \times \bigcup_{j=1}^{q-1} \mathbb{S}^{j-1} \times\left(\bigcup_{i=j+1}^{q} \mathbb{S}^{i-j} \times \mathbb{S}^{(q-i+1) * 2}\right)^{(n+1) / 2}
$$


Using $Y_{i}, f r k_{c}(x)$ can be rewritten as follows.

$$
\begin{aligned}
\operatorname{frk}_{c}(x) & =\operatorname{Pr}\left[\left(J_{1}=J_{0}\right) \mid\left(I_{1}=I_{0}\right) \wedge\left(1 \leq J_{0}<I_{0} \leq q\right)\right] \operatorname{Pr}\left[\left(I_{1}=I_{0}\right) \wedge\left(1 \leq J_{0}<I_{0} \leq q\right)\right] \\
& =(1-\eta(x)) \sum_{i=2}^{q} \sum_{\rho, \mathrm{S}_{(i-)}} \frac{1}{|\mathbb{R}||\mathbb{S}|^{i-1}} Y_{i}^{2}\left(\rho, \mathrm{S}_{(i-)}\right) \quad(\text { see }(28) \text { in Appendix C.1 for details) } \\
& =(1-\eta(x)) \sum_{i=2}^{q} \operatorname{Ex}\left[Y_{i}^{2}\right] \geq \frac{1}{q}\left(\sum_{i=2}^{q} \operatorname{Ex}\left[Y_{i}\right]\right)^{2} \quad \text { (by Jensen's and Hölder's inequality) } \\
& \left.=(1-\eta(x)) \frac{1}{q} \operatorname{acc}(x)^{2} \quad \text { (by definition of } Y_{i} \text { and } \operatorname{acc}(x)\right)
\end{aligned}
$$

By substituting (12) and (13) in (11), we get

$$
\operatorname{frk}(x)=\underset{\mathbb{T}_{(2)}}{\operatorname{Pr}}\left[\left(I_{1}=I_{0}\right) \wedge\left(1 \leq J_{0}<I_{0} \leq q\right) \wedge\left(s_{I_{0}}^{0} \neq s_{I_{0}}^{1}\right)\right] \geq \operatorname{acc}(x)\left(\frac{\operatorname{acc}(x)}{q}(1-\eta(x))-\frac{1}{|\mathbb{S}|}\right)
$$

establishing Claim 1.

The fundamental difference between the argument of Claim 1 and the proof of GF Algorithm in [BN06] is the involvement of the additional index $J$. The only reason that the analysis proceeds as in [BN06] is due to the assumption $J \prec I$. Without this assumption, the argument for Claim 1 would require defining a random variable that takes both the indices into consideration (this is indeed the case in the proof of Lemma 9).

Remark 4. In Claim 1, what we have effectively established is that on $\mathrm{O}_{\{\mathrm{I}, \mathrm{D}\}}$, single augmented forking can be carried out as efficiently as an elementary forking.

The remaining two claims require a random variable $Z_{j}$ that captures a single invocation of $\mathcal{Z}$. Let $Z_{j}: \mathbb{R} \times \mathbb{S}^{j-1} \mapsto[0,1]$, for $1 \leq j \leq q-1$, be defined by setting

$$
Z_{j}\left(\rho, \mathrm{S}_{(j-)}\right)=\operatorname{Pr}\left[\left(J^{\prime}=J=j\right) \wedge\left(I^{\prime}=I\right) \wedge(j<I \leq q) \wedge\left(s_{I}^{\prime} \neq s_{I}\right)\right]
$$

given

$$
\begin{gathered}
\left(\mathrm{S}_{(j I)},\left(\mathrm{S}_{(I+)}, \mathrm{S}_{(I+)}^{\prime}\right)\right) \stackrel{\mathrm{U}}{\leftarrow} \text { and } \\
\left((I, J, \sigma),\left(I^{\prime}, J^{\prime}, \sigma^{\prime}\right)\right) \leftarrow \mathcal{Z}\left(x,\left\{\mathrm{~S}_{(j-)}, \mathrm{S}_{(j I)}, \mathrm{S}_{(I+)}\right\},\left\{\mathrm{S}_{(j-)}, \mathrm{S}_{(j I)}, \mathrm{S}_{(I+)}^{\prime}\right\} ; \rho\right) .
\end{gathered}
$$

A crucial point is that the observation $\mathrm{O}_{\mathrm{I}}$ has been considered in the definition of $Z_{j}$. Without this assumption, the analysis would require a random variable that takes both the indices into consideration (as we do later in the proof of Lemma 10 using a random variable $Z_{i, j}$ ). Briefly, our aim is to bound $m f r k_{3}(x)$ in terms of $Z_{j}$ (Claim 2) and then bound $Z_{j}$ in terms of $f r k(x)$ (Claim 3).

\section{Claim 2.}

$$
m f r k_{3}(x) \geq \frac{1}{q^{(n-1) / 2}}\left(\sum_{j=1}^{q-1} \operatorname{Ex}\left[Z_{j}\right]\right)^{(n+1) / 2}-\frac{(n-1)(n+1)}{8|\mathbb{S}|}\left(\sum_{j=1}^{q-1} \operatorname{Ex}\left[Z_{j}\right]\right)
$$

Argument. The first step is to separate the "core" subevents out of the event E as shown below.

$$
\begin{aligned}
\operatorname{Pr}[\mathrm{E}] & =\operatorname{Pr}\left[\mathrm{B} \wedge\left(\wedge_{k=0,2, \ldots, n-1} \mathrm{C}_{k}\right) \wedge\left(\wedge_{k=2,4, \ldots, n-1} \mathrm{D}_{k}\right)\right] \\
& \geq \operatorname{Pr}\left[\mathrm{B} \wedge \mathrm{C}_{0} \wedge\left(\wedge_{k=2,4, \ldots, n-1} \mathrm{C}_{k} \wedge \mathrm{D}_{k, c}\right)\right]-\operatorname{Pr}\left[\mathrm{B} \wedge \mathrm{C}_{0} \wedge\left(\vee_{k=2,4, \ldots, n-1} \mathrm{D}_{k, s}\right)\right]
\end{aligned}
$$


We denote the first part of (16) by $m f r k_{3, c}(x)$ and the second part by $m f r k_{3, s}(x)$ and analyse them separately.

$$
\begin{aligned}
\operatorname{mfrk}_{3, c}(x) & =\sum_{j=1}^{q-1} \operatorname{Pr}\left[\wedge_{k=0,2, \ldots, n-1}\left(\left(J_{k+1}=J_{k}=j\right) \wedge\left(I_{k+1}=I_{k}\right) \wedge\left(j<I_{k} \leq q\right) \wedge\left(s_{I_{k}}^{k+1} \neq s_{I_{k}}^{k}\right)\right)\right] \\
& =\sum_{j=1}^{q-1} \sum_{\rho, \mathrm{S}_{(j-)}} \frac{\prod_{k=0,2}^{n-1} Z_{j}\left(\rho, \mathrm{S}_{(j-)}\right)}{|\mathbb{R}||\mathbb{S}|^{j-1}} \quad \text { (see (29) in Appendix C.1 for details) } \\
& =\sum_{j=1}^{q-1} \operatorname{Ex}\left[Z_{j}^{(n+1) / 2}\right] \geq \frac{1}{q^{(n-1) / 2}}\left(\sum_{j=1}^{q-1} \operatorname{Ex}\left[Z_{j}\right]\right)^{(n+1) / 2} \quad \text { (by Jensen's and Hölder's inequality) }
\end{aligned}
$$

Using a similar line of approach as above, it is possible to bound $m f r k_{3, s}$ too in terms of $Z_{j}$ (see (31) in Appendix C.1 for the intermediate steps).

$$
\operatorname{mfrk}_{3, s}(x)=\frac{(n-1)(n+1)}{8|\mathbb{S}|}\left(\sum_{j=1}^{q-1} \operatorname{Ex}\left[Z_{j}\right]\right)
$$

Substituting the value of $m f r k_{3, c}(x)$ from (17) and $m f r k_{3, s}(x)$ from (18) in (16) proves Claim 2.

What remains is to relate Claim 1 and Claim 2 by establishing

Claim 3. $\sum_{j=1}^{q-1} E x\left[Z_{j}\right] \geq \operatorname{frk}(x)$.

Argument. Recall that the bound given in Claim 1 for $f r k(x)$ is with the probability taken over the set

$$
\mathbb{T}_{(2)}:=\mathbb{R} \times \bigcup_{i=2}^{q} \mathbb{S}^{i-1} \times \mathbb{S}^{(q-i+1) * 2} .
$$

Let $\mathbb{T}_{(2, j>)}$ denote the underlying set for the random variable $Z_{j}$. From the definition of $Z_{j}$, we can infer that

$$
\begin{aligned}
\mathbb{T}_{(2, j>)}:= & \left(\mathbb{R} \times \mathbb{S}^{j-1} \times \bigcup_{i=j+1}^{q} \mathbb{S}^{i-j} \times \mathbb{S}^{(q-i+1) * 2}\right)=\left(\mathbb{R} \times \bigcup_{i=j+1}^{q} \mathbb{S}^{i-1} \times \mathbb{S}^{(q-i+1) * 2}\right) \text { and } \\
& \operatorname{Ex}\left[Z_{j}\right]=\operatorname{Pr}_{\mathbb{T}_{(2, j>)}}\left[\left(J^{\prime}=J=j\right) \wedge\left(I^{\prime}=I\right) \wedge(j<I \leq q) \wedge\left(s_{I}^{\prime} \neq s_{I}\right)\right] .
\end{aligned}
$$

Notice that the set $\mathbb{T}_{(2, j>)}$ is a subset of the set $\mathbb{T}_{(2)}$. In fact, $\mathbb{T}_{(2)}$ can be partitioned into the two sets $\mathbb{T}_{(2, j<)}$ and $\mathbb{T}_{(2, j>)}$ where

$$
\mathbb{T}_{(2, j<)}:=\mathbb{R} \times \bigcup_{i=2}^{j} \mathbb{S}^{i-1} \times \mathbb{S}^{(q-i+1) * 2} .
$$

At this point the subtle change in the definition of the $\operatorname{acc}(x)$ comes into play. The accepting condition had been altered ${ }^{13}$ from $(I \geq 1) \wedge(J \geq 1)$ in Lemma 1 to $(1 \leq J<I \leq q)$ in Lemma 2. By implication

$$
\operatorname{Pr}_{\mathbb{T}_{(2, j<)}}\left[\left(J^{\prime}=J=j\right) \wedge\left(I^{\prime}=I\right) \wedge(j<I \leq q) \wedge\left(s_{I}^{\prime} \neq s_{I}\right)\right]=0 .
$$

\footnotetext{
${ }^{13}$ To be precise, we have taken into account the definition of $\mathcal{Y}$ while defining $\operatorname{acc}(x)$. Interestingly, [BPW12] could also have incorporated this.
} 
Intuitively speaking, (20) is a consequence of the fact that the definition of $\operatorname{acc}(x)$ prohibits a successful $\mathcal{Y}$ from returning $(I, J)$ such that $I \leq J$. The fact that $\mathbb{T}_{(2, j>)} \subset \mathbb{T}_{(2)}$, in conjunction with (20) implies

$$
\begin{aligned}
& \operatorname{Pr}_{\mathbb{T}_{(2, j>)}}\left[\left(J^{\prime}=J=j\right) \wedge\left(I^{\prime}=I\right) \wedge(j<I \leq q) \wedge\left(s_{I}^{\prime} \neq s_{I}\right)\right] \geq \\
& \quad \underset{\mathbb{T}_{(2)}}{\operatorname{Pr}}\left[\left(J^{\prime}=J=j\right) \wedge\left(I^{\prime}=I\right) \wedge(j<I \leq q) \wedge\left(s_{I}^{\prime} \neq s_{I}\right)\right] .
\end{aligned}
$$

Finally, on taking the sum of $\operatorname{Ex}\left[Z_{j}\right]$ over the index $j$, we get

$$
\begin{aligned}
\sum_{j=1}^{q-1} \operatorname{Ex}\left[Z_{j}\right] & \geq \sum_{j=1}^{q-1} \operatorname{Pr}_{(2)}\left[\left(J^{\prime}=J=j\right) \wedge\left(I^{\prime}=I\right) \wedge(j<I \leq q) \wedge\left(s_{I} \neq s_{I}^{\prime}\right)\right] \quad \text { (using (21)) } \\
& =\operatorname{Pr}_{(2)}^{\operatorname{Pr}}\left[\left(J^{\prime}=J\right) \wedge\left(I^{\prime}=I\right) \wedge(1 \leq J<I \leq q) \wedge\left(s_{I} \neq s_{I}^{\prime}\right)\right]=f r k(x)
\end{aligned}
$$

completing the argument.

On putting all the three claims together, we get

$$
\begin{aligned}
m \operatorname{frk}_{3}(x) & \geq \frac{\operatorname{frk}(x)^{(n+1) / 2}}{q^{(n-1) / 2}}-\frac{(n-1)(n+1)}{8|\mathbb{S}|} \operatorname{frk}(x) \\
& =\operatorname{frk}(x) \cdot\left(\frac{\operatorname{frk}(x)^{(n-1) / 2}}{q^{(n-1) / 2}}-\frac{(n-1)(n+1)}{8|\mathbb{S}|}\right)
\end{aligned}
$$

Finally, taking the expectation over $x \stackrel{\$}{\leftarrow} \mathcal{G}_{I}$, yields

$$
f r k \geq \operatorname{acc}\left(\frac{\operatorname{acc}}{q}(1-\eta)-\frac{1}{|\mathbb{S}|}\right) \text { and } m f r k_{3} \geq f r k \cdot\left(\frac{f r k^{(n-1) / 2}}{q^{(n-1) / 2}}-\frac{(n-1)(n+1)}{8|\mathbb{S}|}\right)
$$

establishing Lemma 2. We conclude with the comment that on assuming $|\mathbb{S}| \gg 1$, one gets $m f r k_{3} \approx a c c^{n+1} / q^{n}$.

\section{Revisiting the Security Argument of Existing Protocols}

We now take a closer look at the protocols that employ the MF Algorithm in their security argument. Our primary objective is to examine the applicability of the observations $\mathrm{O}_{\mathrm{I}}$ and $\mathrm{O}_{\mathrm{D}}$. We also comment on the design of the corresponding wrappers. We use the proxy signature scheme of Boldyreva et al. (BPW-PSS) [BPW12] to motivate the notion of (random oracle) dependence and show how both $\mathrm{O}_{I}$ and $\mathrm{O}_{\mathrm{D}}$ can be captured in the security argument. We then suggest a small modification-coined "binding"-in the identity-based signature scheme of Galindo and Garcia (GG-IBS) [GG09] to induce hash function dependence and briefly comment on its security (a detailed argument is provided in Appendix E). The effect on zero-knowledge protocol of Chow et al. (CMW-ZKP) [CMW12] is discussed in $\S 4.4$.

\subsection{Random-Oracle Dependence}

The notion of hash function (or, random-oracle) dependence can be best appreciated through a concrete example. Consider the construction of BPW-PSS scheme (see Figure 5 of Appendix D.1). The protocol uses three hash functions: $G, R$ and $H$. The hash functions are called by the different algorithms in a certain order and here we'll mainly focus on the generation of proxy signature. Observe that Delegation uses $G$ for producing proxy certificates and 
$\mathrm{R}$ for generating proxy secret keys; $\mathrm{H}$ is used in Proxy Signing for computing proxy signatures. The proxy signature is computed using the proxy secret key which, in turn, is computed using the proxy certificate. Hence, to generate a proxy signature the hash function calls must follow a logical order: $\mathrm{G}<\mathrm{R}<\mathrm{H}$ (here $<$ denotes 'followed by').

Now let's take a look at the structure of hash function calls:

$$
c:=\mathrm{G}(0\|S\| Y) \quad r:=\mathrm{R}(S\|Y\| c) \quad h:=\mathrm{H}(0\|\tilde{m}\| S\|Y\| V \| r) .
$$

The critical point is to observe the binding between the hash functions. $\mathrm{R}$ takes as an input $c$ which is the output of $\mathrm{G}$; $\mathrm{H}$ takes as an input $r$ which is the output of R. Consequently, to produce a proxy signature, one has to (except, with a negligible probability of guessing) call the hash functions in the order: $\mathrm{G}<\mathrm{R}<\mathrm{H}$ (which is also the logical order). In other words, the logical order has been explicitly imposed as the only viable order.

Next, consider the simulation of the protocol environment for the BPW-PSS where the hash functions are modelled as random oracles. The aforesaid order among the hash functions naturally translates into an order among the corresponding random oracles. Hence, to forge a proxy signature, an adversary has to (except, with a negligible probability of guessing) make the target random oracle queries in the order $\mathrm{G}<\mathrm{R}<\mathrm{H}$. In other words, if $K, J$ and $I$ are the indices that refer to the target $\mathrm{G}, \mathrm{R}$ and $\mathrm{H}$ random oracle queries corresponding to the forgery, then it follows that $(1 \leq K<J<I \leq q)$. Now, consider a second round of simulation of the adversary initiated by a forking at $I$ (corresponding to the successful target H-query). Suppose the adversary is successful in the second round and, in addition, the target $\mathrm{H}$ index for the second round matches with the first (i.e., $I^{\prime}=I$ ). It is not difficult to see that, due to the binding between the random oracles, the $\mathrm{R}$ and $\mathrm{G}$ target indices for the two rounds also have to (except, as we shall see, with probability denoted by $\eta_{b}$ ) match. The advantage with which an adversary can forge a proxy signature on violating this condition is, in fact, (asymptotically) negligible. Hence, $\left(I^{\prime}=I\right)$ implies $\left(J^{\prime}=J\right)$ and $\left(K^{\prime}=K\right)$. We say that the random oracle $\mathrm{H}$ is "dependent" on the random oracles $\mathrm{G}$ and $\mathrm{R}$ (denoted by $\{\mathrm{G}, \mathrm{R}\} \prec \mathrm{H}$ ) over these two rounds (in other words, within the wrapper of $\mathcal{Z}$ in the context of multiple forking). Using a similar line of argument, one can establish that $\mathrm{R}$ is dependent on $\mathrm{G}$ (i.e. $\mathrm{G} \prec \mathrm{R}$ ). On putting together the two observations, in the case of BPW-PSS we get $G \prec R \prec H$. A little more formally, the dependence among two random oracles is defined as follows.

Definition 2 (Random-Oracle Dependence). Consider the oracle replay attack in the context of a cryptographic protocol that employs two hash functions $\mathrm{H}_{1}$ and $\mathrm{H}_{2}$ modelled as random oracles. Let $J$ [resp. I] denote the target $\mathrm{H}_{1}$-index [resp. $\mathrm{H}_{2}$-index] for the first round of simulation of the protocol. Also, let $J^{\prime}$ [resp. $I^{\prime}$ ] denote the target $\mathrm{H}_{1}$-index [resp. $\mathrm{H}_{2}$-index] for the second round of simulation that was initiated by a forking at $I$. Suppose that the adversary was successful in both the rounds. The random oracle $\mathrm{H}_{2}$ is defined to be $\eta$-dependent on the random oracle $\mathrm{H}_{1}$ on the target query (denoted by $\mathrm{H}_{1} \prec \mathrm{H}_{2}$ ) if the following criteria are satisfied: i) $(1 \leq J<I \leq q)$ or, in other words $\mathrm{H}_{1}<\mathrm{H}_{2}$; and ii) $\operatorname{Pr}\left[\left(J^{\prime} \neq J\right) \mid\left(I^{\prime}=I\right)\right] \leq \eta{ }^{14}$

The second criterion, in other words, requires $\left(I^{\prime}=I\right) \Longrightarrow\left(J^{\prime}=J\right)$ with probability at least $(1-\eta)$; for the criterion to hold with overwhelming probability, $\eta$ should be negligible in $\kappa$. A logical order among the "hash" functions in the protocol does not necessarily mean that there is a dependence among them. ${ }^{15}$ Hence, one may need to impose explicit dependence among the hash functions. A natural way to induce the dependence $\mathrm{H}_{1} \prec \mathrm{H}_{2}$ is through the binding technique used in BPW-PSS: by making the input to $\mathrm{H}_{2}$ a function of $\mathrm{H}_{1}$ 's output.

\footnotetext{
${ }^{14}$ Note that the second criterion is the concrete instance of index-dependence in Definition 1. The first criterion, on the other hand, has been already absorbed in the corresponding definition of $\mathcal{Y}$ (see Condition $\mathrm{B}$ of $\mathbb{A}_{2}$ and $\mathbb{A}_{3}$ ).

${ }^{15}$ Dependence is induced by design in BPW-PSS and was not present in the original GG-IBS. On the other hand, dependence for the CMW-ZKP follows from the logical order.
} 
Claim 4 (Binding induces dependence). Consider the hash functions (and the corresponding random oracles) described in Definition 2. Let $q_{1}$ denote the upper bound on the number of queries to the random oracle $H_{1}$. In addition, let $\mathbb{R}_{1}$ denote the range of $H_{1}$. Binding $H_{2}$ to $H_{1}$ (by making the input to $\mathrm{H}_{2}$ a function of $H_{1}$ 's output) induces a random-oracle dependence $H_{1} \prec H_{2}$ with $\eta_{b}:=q_{1}\left(q_{1}-1\right) /\left|\mathbb{R}_{1}\right|$.

Argument. Suppose $\left(I^{\prime}=I\right)$ but $\left(J^{\prime} \neq J\right)$. It is not difficult to see that this can happen only in the scenario illustrated in Figure 3. That is, $i$ ) the adversary made a query $Q_{J^{*}}$ (to the random oracle $\mathrm{H}_{1}$ ) that is different from $\mathrm{Q}_{J}$, the target $\mathrm{H}_{1}$-query for the round 0 of simulation; and ii) $\mathrm{Q}_{J^{*}}$ was also responded to with $s_{J}$ (the simulator's response to $\mathrm{Q}_{J}$ ). However, this is tantamount to

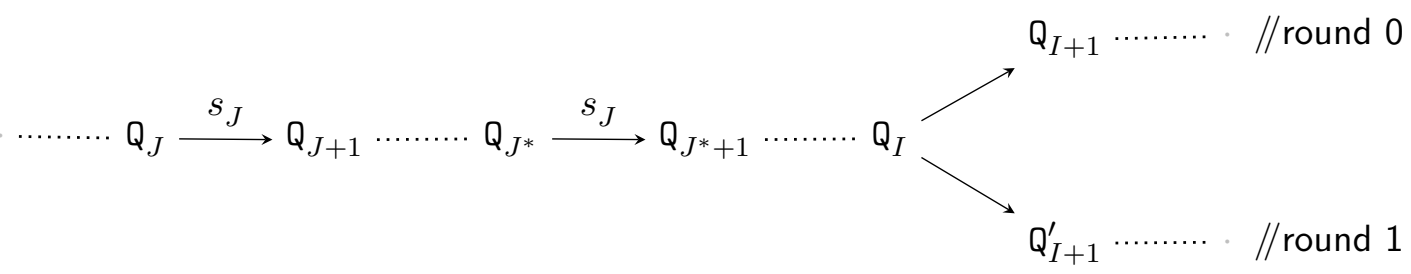

Figure 3: Violation of random-oracle dependence.

a collision on the random function corresponding to the oracle $\mathrm{H}_{1}$ and according to the birthday bound, can happen with probability at most $q_{1}\left(q_{1}-1\right) /\left|\mathbb{R}_{1}\right|$.

Remark 5. The notion of random-oracle dependence can be naturally adapted for (interactive) commitment-challenge rounds (through [FS87]) with the notion of target commitmentchallenge round in place of the notion of target random oracle query. This is demonstrated for the CMW-ZKP scheme in coming section ( $\$ 4.4)$.

\subsection{The Boldyreva-Palacio-Warinschi Proxy Signature Scheme}

We refer the reader to [BPW12] for the definition of proxy signature and the original security argument of BPW-PSS (the construction is reproduced in Appendix D.1). The extremely technical and long security argument of [BPW12] consists of five reductions $\mathcal{B}$ through $\mathcal{F}$, with the associated wrappers $\mathcal{Y}, \mathcal{Z}^{\prime}, \mathcal{U}, \mathcal{V}$ and $\mathcal{W}$ respectively ${ }^{16}$. The discrete-log problem is reduced to breaking the scheme in each of them. The reductions $\mathcal{C}, \mathcal{D}$ and $\mathcal{F}$ use the MF Algorithm, whereas, the reductions $\mathcal{B}$ and $\mathcal{E}$ use the GF Algorithm. Some features of the relevant reductions $\mathcal{C}, \mathcal{D}$ and $\mathcal{F}$ are summarised in Table 4 .

Inherent dependence and wrapper design. We have already pointed out the inherent dependence of the hash functions used in BPW-PSS. This inbuilt dependence among the hash functions, along with a careful design of the wrappers, ensures that the MF Algorithm is applied properly. An integral part in the design of the wrappers is the explicit check for the logical order among the target random oracle queries. If the order is violated, the wrapper sets a flag and returns $(0, \perp)$. For example, consider the reduction $\mathcal{C}$ and the associated wrapper $\mathcal{Z}^{\prime}$ from [BPW12]. The check ensures that the indices $J$ and $I$ (that the wrapper returns) always correspond to the target query for the random oracles $\mathrm{R}$ and $\mathrm{H}$ respectively. Moreover, due to dependence, the adversary is bound (except with a negligible probability of guessing) to make the target oracle queries in the logical order, i.e. $\mathrm{R}$ followed by $\mathrm{H}$. These two factors ensure that the reduction $\mathcal{C}$ will end up with a correct solution to the DLP whenever the MF Algorithm is successful. The same strategy has been followed meticulously in the construction of $\mathcal{D}$ and

\footnotetext{
${ }^{16}$ We have renamed the wrapper $\mathcal{Z}$ in [BPW12] to $\mathcal{Z}^{\prime}$ for avoiding confusion with the logical wrapper $\mathcal{Z}$ used in the analysis.
} 
$\mathcal{F}$ as well. So the notion of index dependence is to some extent used implicitly in the security argument of BPW-PSS.

However, when we come to the MF Algorithm that corresponds to this particular scheme, neither the notion of index dependence nor that of independence between the logical wrapper $\mathcal{Z}$ is considered in the analysis. This brings us to the improved security argument.

\subsubsection{Improved Security Argument}

The new security argument takes advantage of both the observations $\mathrm{O}_{\mathrm{I}}$ and $\mathrm{O}_{\mathrm{D}}$. We have already seen that $\mathrm{O}_{\mathrm{D}}$ is applicable due to the existing binding. As for $\mathrm{O}_{\mathrm{I}}$, we again consider the case of reduction $\mathcal{C}$ and its wrapper $\mathcal{Z}^{\prime}$ due to their relative simplicity. A similar argument works for reductions $\mathcal{D}$ and $\mathcal{F}$ as well.

$\mathcal{Z}^{\prime}$ is designed to output index $I$ (resp. $J$ ) corresponding to the target $\mathrm{H}$ (resp. R) query. $\mathcal{C}$ uses the MF Algorithm $\mathcal{M}_{\mathcal{Z}^{\prime}, 3}$ to secure a set of four forgeries $\sigma:=(z, h, r), \hat{\sigma}:=(\hat{z}, \hat{h}, \hat{r}), \bar{\sigma}:=$ $(\bar{z}, \bar{h}, \bar{r})$ and $\dot{\sigma}:=(\dot{z}, \dot{h}, \dot{r})$ with

$$
\begin{array}{ll}
z=v+\left(r \alpha+y+c \log _{g} \mathrm{pk}_{i}\right) h & \bar{z}=\bar{v}+\left(\bar{r} \alpha+y+c \log _{g} \mathrm{pk}_{i}\right) \bar{h} \\
\hat{z}=v+\left(r \alpha+y+c \log _{g} \mathrm{pk}_{i}\right) \hat{h} & \dot{z}=\bar{v}+\left(\bar{r} \alpha+y+c \log _{g} \mathrm{pk}_{i}\right) \dot{h} .
\end{array}
$$

What we have now is a system of four congruences in four (effective) unknowns $\{\alpha,(y+$ $\left.\left.c \log _{g} \mathrm{pk}_{i}\right), v, \bar{v}\right\}$ with $\alpha$ being the solution to the DLP. The forgeries $\sigma$ and $\hat{\sigma}$ (resp. $\bar{\sigma}$ and $\dot{\sigma}$ ) can be clubbed together as they constitute the output of the the logical wrapper $\mathcal{Z}$ that is associated to wrapper $\mathcal{Z}^{\prime}$ of $\mathcal{M}_{\mathcal{Z}^{\prime}, 3}$. From the structure of the $\mathrm{H}$ query, it follows that the index $I$ corresponds to the unknown $v$. The process of solving for $\alpha$ starts by eliminating the unknown $v$ from each of $\mathcal{Z}$ s (i.e., $v$ from $(z, \hat{z})$ and $\bar{v}$ from $(\bar{z}, \dot{z}))$. What is necessary at this point is that the $I$ indices must match within $\mathcal{Z}$. The solution is not affected by the value of $I$ in the second invocation of $\mathcal{Z}$. In other words, eliminating $v$ from $(z, \hat{z})$ is not affected by the pair $(\bar{z}, \dot{z})$ and vice versa. Hence, from the point of view of the reduction, it doesn't make any difference whether we relax the condition to accommodate independence (the system of congruences one ends up with is exactly the same as in (23)). In fact, the reduction is unlikely to achieve anything by restricting the indices. Hence, the independence of the $I$ indices is applicable.

Remark 6. The notion of independence can be better appreciated if we visualise the process of multiple forking in terms of congruences and unknowns. At a high level, what the reduction algorithm secures from the MF Algorithm is a set of $n+1$ congruences in $n+1$ unknowns (for some odd $n$ ). One of these unknowns is the solution to the hard problem that the reduction wants to solve. The MF Algorithm needs to ensure that the congruences are linearly independent of each other with a certain non-negligible probability. The claim in $\mathrm{O}_{\mathrm{I}}$ can then be restated as: even if the condition on the $I$ indices is relaxed as in $\mathrm{O}_{\mathrm{I}}$, we still end up with a system of $n+1$ congruences in $n+1$ unknowns.

To sum it up, in order to harness both $\mathrm{O}_{\mathrm{I}}$ and $\mathrm{O}_{\mathrm{D}}$, the only change in the security argument of [BPW12] is to use the GMF Algorithm $\mathcal{N}_{\mathbb{A}_{3}, \mathcal{Y}, n}$ (with Lemma 2) instead of the original MF Algorithm. The resulting changes are summarised in Table 4.

\subsection{The Galindo-Garcia Identity-Based Signature}

The original construction from [GG09] is reproduced in Figure 6 of Appendix D.2 and we refer the reader to [BNN04] for the definition and security model of IBS schemes. Two hash functions are used in the scheme: $\mathrm{H}$ and $\mathrm{G}$ (both map arbitrary length strings to the set $\mathbb{Z}_{p}^{*}$ ). The structure of hash function calls is given below.

$$
c:=\mathrm{H}(R \| \mathrm{id}) \quad d:=\mathrm{G}(\mathrm{id}\|A\| m)
$$




\begin{tabular}{|c|c|c|c|c|}
\hline \multirow{2}{*}{ Security Argument } & \multicolumn{4}{|c|}{ Particulars } \\
\cline { 2 - 6 } & Reduction & $\mathcal{C}$ & $\mathcal{D}$ & $\mathcal{F}$ \\
\cline { 2 - 6 } & Oracles involved & $\mathrm{R}$ and $\mathrm{H}$ & $\mathrm{G}$ and $\mathrm{H}$ & $\mathrm{G}$ and $\mathrm{H}$ \\
\hline \multirow{2}{*}{ Original } & Forking Algorithm & $\mathcal{M}_{\mathcal{Z}^{\prime}, 3}$ & $\mathcal{M}_{\mathcal{U}, 5}$ & $\mathcal{M}_{\mathcal{W}, 5}$ \\
\cline { 2 - 6 } & Degradation & $\mathrm{O}\left(\left(q_{\mathrm{R}}+q_{\mathrm{H}}\right)^{6}\right)$ & $\mathrm{O}\left(\left(q_{\mathrm{G}}+q_{\mathrm{H}}\right)^{10}\right)$ & $\mathrm{O}\left(\left(q_{\mathrm{R}}+q_{\mathrm{H}}\right)^{10}\right)$ \\
\hline \multirow{2}{*}{ Improved } & Forking Algorithm & $\mathcal{N}_{\mathbb{A}_{3}, \mathcal{Z}^{\prime}, 3}$ & $\mathcal{N}_{\mathbb{A}_{3}, \mathcal{U}, 5}$ & $\mathcal{N}_{\mathbb{A}_{3}, \mathcal{W}, 5}$ \\
\cline { 2 - 6 } & Degradation & $\mathrm{O}\left(\left(q_{\mathrm{R}}+q_{\mathrm{H}}\right)^{3}\right)$ & $\mathrm{O}\left(\left(q_{\mathrm{G}}+q_{\mathrm{H}}\right)^{5}\right)$ & $\mathrm{O}\left(\left(q_{\mathrm{R}}+q_{\mathrm{H}}\right)^{5}\right)$ \\
\hline
\end{tabular}

Table 4: Comparison of the original and improved security argument for BPW-PSS. $q_{\mathrm{G}}, q_{\mathrm{R}}$ and $q_{\mathrm{H}}$ denote the upper bound on the respective hash oracle queries.

$\mathrm{H}$ is used to generate the user secret key which, in turn, is required to sign on a message (using $\mathrm{G})$. Hence $\mathrm{H}<\mathrm{G}$ constitutes the logical order for the hash functions. However, no binding is in place by construction. The absence of binding (and consequently, the absence of dependence) is precisely the reason for the "incompleteness" of the original security argument that was pointed out in [CKK13]. The incompleteness was addressed in [CKK13] by using the two-reduction strategy- give separate reductions for each of the orders of the target $\mathrm{H}$ and $\mathrm{G}$ calls.

\subsubsection{Modified Galindo-Garcia IBS}

A better alternative to the two-reduction fix is to enforce the logical order on the adversary by binding the G-oracle to the H-oracle. ${ }^{17}$ A modified GG-IBS with the aforesaid binding is given in Appendix D.3. In short, the structure of the hash function call to $G$ to something like the following:

$$
d:=\mathrm{G}(\mathrm{id}\|A\| m \| c) \quad \text { where } \quad c:=\mathrm{H}(R \| \text { id }) .
$$

Once the binding is in place, the adversary (except with a negligible probability) is bound to make the target queries in the logical order. In addition, through an argument similar to that of BPW-PSS, one can show that $\mathrm{O}_{\mathrm{I}}$ is also applicable. Accordingly, the security argument for the modified GG-IBS consists of two reductions $\mathcal{R}_{1}^{\prime}$ and $\mathcal{R}_{3}^{\prime}$. The core of these reductions remains the same as in $\mathcal{R}_{1}$ and $\mathcal{R}_{3}$ respectively of [CKK13]. The only major change is the use of GMF Algorithm $\mathcal{N}_{\mathbb{A}_{3}, \mathcal{Y}, n}$ (with Lemma 2) in $\mathcal{R}_{3}^{\prime}$ instead of the original MF Algorithm in $\mathcal{R}_{3}$. For the sake of completeness, we give a detailed description of $\mathcal{R}_{3}^{\prime}$ (including the wrapper) in Appendix E. The overall effect is summarised in Table 5.

\subsection{The Chow-Ma-Weng ZKP for Simultaneous Discrete Logarithms}

We confine ourselves to the basic protocol $(n=2)$ which is reproduced in Figure 8 of Appendix D.4. The argument can be easily extended for arbitrary values of $n$. There are two objects in consideration: the hash function $\mathrm{H}$ and the (interactive) commitment-challenge round ${ }^{18}$

\footnotetext{
${ }^{17}$ A noteworthy observation is that, binding the H-oracle to the G-oracle-i.e., setting up the dependence $\mathrm{G} \prec \mathrm{H}$ instead of $\mathrm{H} \prec \mathrm{G}$-in the GG-IBS allows more efficient reductions to DLP (using general forking). However, this disturbs the logical order of the hash functions. In such a protocol, the PKG will have to issue user secret keys for each message to be signed, rendering it impractical.

${ }^{18}$ The round of interaction can be replaced with a hash function (also denoted by $\mathrm{C}$ ) to make the protocol noninteractive [FS87].
} 


\begin{tabular}{|c|c|c|c|c|}
\hline \multirow{2}{*}{ Scheme } & \multicolumn{4}{|c|}{ Security Argument } \\
\hline \multirow{2}{*}{ GG-IBS [CKK13] } & Reduction & $\mathcal{R}_{1}$ & $\mathcal{R}_{2}$ & $\mathcal{R}_{3}$ \\
\cline { 2 - 6 } & Forking Algorithm & $\mathcal{F}_{\mathcal{Y}}$ & $\mathcal{M}_{\mathcal{Y}, 1}$ & $\mathcal{M}_{\mathcal{Y}, 3}$ \\
\cline { 2 - 6 } & Degradation & $\mathrm{O}\left(q_{\mathrm{G}} q_{\varepsilon}\right)$ & $\mathrm{O}\left(\left(q_{\mathrm{H}}+q_{\mathrm{G}}\right)^{2}\right)$ & $\mathrm{O}\left(\left(q_{\mathrm{H}}+q_{\mathrm{G}}\right)^{6}\right)$ \\
\hline \multirow{2}{*}{ Modified GG-IBS (Figure 6) } & Reduction & $\mathcal{R}_{1}^{\prime}$ & \multicolumn{2}{|c|}{$\mathcal{R}_{3}^{\prime}$} \\
\cline { 2 - 6 } & Forking Algorithm & $\mathcal{F}_{\mathcal{Y}}$ & \multicolumn{2}{|c|}{$\mathcal{N}_{\mathbb{A}_{3}, \mathcal{Y}, 3}$} \\
\cline { 2 - 5 } & Degradation & $\mathrm{O}\left(q_{\mathrm{G}} q_{\varepsilon}\right)$ & $\mathrm{O}\left(\left(q_{\mathrm{H}}+q_{\mathrm{G}}\right)^{3}\right)$ \\
\hline
\end{tabular}

Table 5: Degradation for Galindo-Garcia IBS and its variant with binding. $q_{\mathrm{G}}$ and $q_{\mathrm{H}}$ denote the upper bound on the respective hash oracle queries, whereas, $q_{\varepsilon}$ denotes upper bound on the extract queries. We have assumed $\eta$ to be negligible.

which we denote by C. The soundness of the CMW-ZKP is based on the hardness of the DLP: the reduction, denoted by $\mathcal{B}$, uses the (original) $\mathrm{MF}$ Algorithm $\mathcal{M}_{\mathcal{Y}, 5}$ to launch a nested replay attack involving $\mathrm{H}$ and $\mathrm{C}$.

Lemma 5 (Soundness, Lemma 4 in [CMW12]). In the random-oracle model (the hash function $H$ will be modelled as a random oracle), if there exists an adversary $\mathcal{A}$ that can $\epsilon$-break the soundness of the CMW protocol (i.e. $\mathcal{V}$ accepts but $\log _{g} y_{1} \neq \log _{g} y_{2}$ ), there exists an algorithm $\mathcal{B}$ which can $\epsilon^{\prime}$-solve the DLP with

$$
\epsilon^{\prime} \geq \epsilon \cdot\left(\frac{\epsilon^{6}}{\left(q_{H}+q_{C}\right)^{10}}-\frac{5}{p}\right)
$$

where $q_{H}$ is the number of random oracle query made by $\mathcal{A}$ and $q_{C}$ is the number of interactions between $\mathcal{A}$ and $\mathcal{B} .^{19}$

The notion of binding/dependence for the CMW-ZKP is, interestingly (and contrary to the previous two examples), between a random oracle $(\mathrm{H})$ and an interactive commitmentchallenge round (C).

\subsubsection{A Case for (In)Dependence}

We now elaborate on the aspects of dependency and independence in the context of CMW protocol (see Figure 8).

Condition $\mathrm{O}_{\mathrm{I}}$. Recall that the commitment $v$ is of the form $\left(g^{z} h\right)^{k}$, where $z:=\mathrm{H}\left(y_{1}, y_{2}\right)$. By construction, the prover has to compute the value of $z$ before making the commitment and the verifier returns the challenge $c$ only after receiving the commitment. Hence the logical order of $\mathrm{H}<\mathrm{C}$. However, it also results in a natural binding between $\mathrm{C}$ and $\mathrm{H}$ which leads to the dependence $\mathrm{H} \prec \mathrm{C} .{ }^{20}$ Next, we consider the simulation of the protocol, in particular, the first invocation of $\mathcal{Z}$. At the end of round 0 , the adversary produces a cheating transcript $\left(v_{1}^{1}, c_{1}^{1}, s_{1}^{1}\right)$,

\footnotetext{
${ }^{19}$ We correct a small error in the original expression: the degradation should be by a factor of $\left(q_{\mathrm{H}}+q_{\mathrm{C}}\right)^{10}$ instead of $\left(q_{\mathrm{H}} \cdot q_{\mathrm{C}}\right)^{10}$.

${ }^{20}$ Let's consider a resource constrained variant of the verifier which, instead of picking the challenge upon receiving a commitment, picks all of the challenges beforehand. From the point of view of the prover, the change is purely conceptual. However, the aforesaid logical order, and also the dependence induced by that logical order, no longer holds.
} 
where $v_{1}^{1}:=\left(g^{z_{1}} h\right)^{k_{1}}$. Let $\left(I_{0}, J_{0}\right)$ be the target indices with $J_{0}$ corresponding to the H-oracle output $z_{1}$ and $I_{0}$ to the commitment $v_{1}^{1}$. round 1 involves forking the adversary at $I_{0}$ and let's assume that, at the end of it, the adversary produces another cheating transcript. If we follow the success conditions of the original MF Algorithm, then this particular forking is successful with probability roughly $1 / q^{2}$ because the cheating transcript has to be on the same commitment $v_{1}^{1}$ (i.e. $I_{1}=I_{0}$ ) and, also, on the same H-oracle output $z_{1}$ (i.e. $J_{1}=J_{0}$ ).

However, it is easy to observe that, due to the natural binding discussed above, an adversary cheating on the commitment $v_{1}^{1}$ (at the end of round 1 ) has to (except, with a negligible probability of guessing) cheat using the H-oracle output of $z_{1}$. In other words, the adversary commits to $z_{1}$ indirectly through $v_{1}^{1}$. Hence, $\left(I_{1}=I_{0}\right)$ has to imply $\left(J_{1}=J_{0}\right)$ and, as a consequence $\mathrm{H} \prec \mathrm{C}$. The same argument holds for the other two invocations of $\mathcal{Z}$ as well.

Condition $\mathrm{O}_{\mathrm{D}}$. The line of argument is basically similar to the one adopted for the BPW-PSS. Consider the first invocation of $\mathcal{Z}$ in the simulation. For the reduction to successfully solve the DLP, the adversary, over these two rounds, has to produce two cheating transcripts on the same commitment (i.e. $I_{1}=I_{0}$ ). This applies to the rounds round $\mathrm{k}$ and round $\mathrm{k}+1$, for $k=2$ and $k=4$ as well. However, it is not required that the $I$ indices should match across these rounds (i.e. $\left(I_{4}=I_{2}=I_{0}\right)$ ). To see this, consider the effect of relaxing the condition on the simulation which is shown in Figure 4.

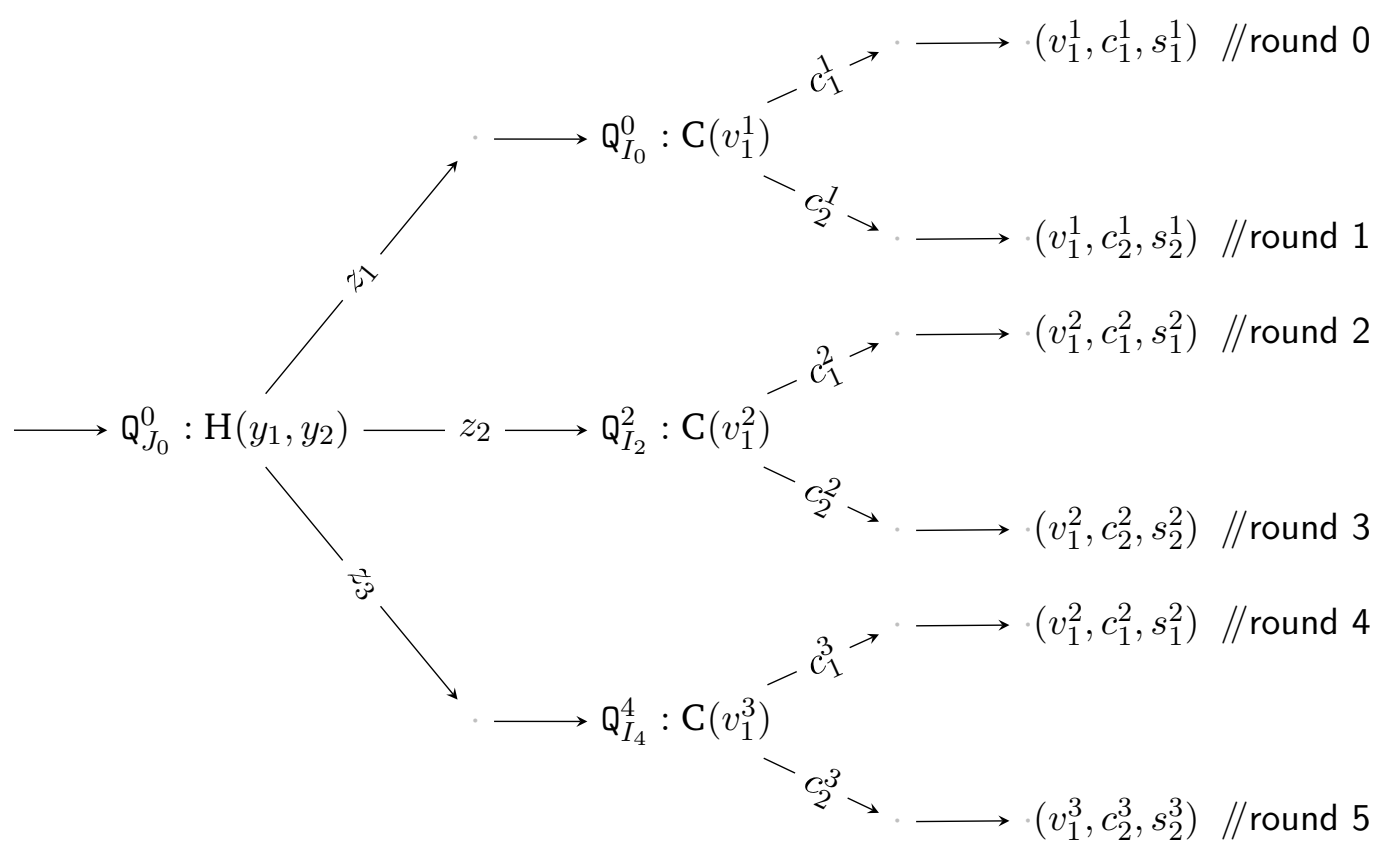

Figure 4: A successful nested replay attack on a CMW-ZKP adversary using $\mathcal{N}_{\mathbb{A}_{3}, \mathcal{Y}, 5}$.

Even though the $I$ indices across $\mathcal{Z}$ do not match $\left(I_{0}, I_{2}\right.$ and $\left.I_{4}\right)$, the set of cheating transcripts that the reduction obtains is still of the form $\left\{\left(v_{1}^{i}, c_{1}^{i}, s_{1}^{i}\right),\left(v_{1}^{i}, c_{2}^{i}, s_{2}^{i}\right)\right\}$, for $i:=1,2,3$. The technique used to solve the DLP in (1) still works. Hence, it suffices that $I$ for the cheating transcripts within the two rounds of a particular invocation of $\mathcal{Z}$ match, but not necessarily across the $\mathcal{Z}$ s.

\subsubsection{Improved Argument}

Before commenting on the improved argument, we would like to point out some issues with the design of the wrapper. The authors have made the following observation: 
"The algorithm $\mathcal{Y}$ here is a wrapper that takes as explicit input the answers from the random oracle $\mathrm{H}$ and the random challenges given by $\mathcal{B}$, calls $\mathcal{A}$ and returns its output together with two integers $I, J$. One of the integers is the index of $\mathcal{A}$ 's calls to the random oracle $\mathrm{H}(\cdot, \cdot)$ and the other is the index of the challenge corresponding to the cheat given by $\mathcal{A}$. ."[emphasis added]

Notice that the correspondence between the indices $(I, J)$ and the target $\mathrm{H}$-oracle call and Cround is not clearly spelt out. The reduction, however, proceeds to solve the DLP under the (implicit) assumption that the wrapper returns a $J$ (resp. $I$ ) which refers to the target H-oracle query (resp. $\mathrm{C}$ round). If the correspondence is reversed, the reduction will end up computing an incorrect solution to the DLP (see Remark 7). To avoid any ambiguity, we emphasise that the wrapper should be explicitly designed to return $I$ (resp. $J$ ) which refers to the target Cround (resp. H-query). Now, the MF Algorithm $\mathcal{M}_{\mathcal{Y}, 5}$ in the original security argument can be replaced with the GMF Algorithm $\mathcal{N}_{\mathbb{A}_{3}, \mathcal{Y}, 5}$ (with Lemma 2) resulting in the following lemma:

Lemma 6 (Soundness). In the random-oracle model, if there exists an adversary $\mathcal{A}$ that can $\epsilon$ break the soundness of the CMW protocol, there exists an algorithm $\mathcal{B}$ which can $\epsilon^{\prime}$-solve the DLP with

$$
\epsilon^{\prime}=\Omega\left(\frac{\epsilon^{6}}{\left(q_{H}+q_{C}\right)^{5}}\right)
$$

where $q_{H}$ is the number of random oracle query made by $\mathcal{A}$, while $q_{C}$ is the number of interactions between $\mathcal{A}$ and $\mathcal{B}$.

Remark 7 (On importance of proper wrapper-design). Consider the simulation of the "resource constrained" verifier that we had discussed in Footnote 20. Upon receiving the values $\left\{s_{1}, \ldots, s_{q}\right\}$ as parameters, the wrapper is designed to fix $\left\{s_{1}, \ldots, s_{q_{c}}\right\}$ as its random challenges before proceeding with the actual simulation. This leads to the wrapper always returning a $J$ index that refers to the target C-round (and an $I$ index that refers to the target H-query). The "artificial" design of the wrapper has the same effect as that of an adversary for the CMW-ZKP making the target calls in the wrong order. Hence, in spite of the simulation being faithful (from the standpoint of the adversary) and the MF Algorithm returning success, the strategy adopted for solving DLP will fail. Thus, it is not difficult to get the design of the wrapper wrong.

\section{Conclusion}

In this paper we have proposed a general framework for the application of the Multiple Forking Lemma. The framework and the corresponding algorithm is derived based on a careful analysis of the original Multiple Forking Lemma and its application in the security argument of various schemes. We prove that our notions of (in)dependence significantly improve upon the bound of the original Lemma. We also show that all known instances of the application of the original Multiple Forking Lemma satisfy the notion of (in)dependence and hence benefit from our improved bound. Whether the new bounds in the security arguments are optimal or can be improved further will be an interesting open question from a theoretical perspective.

\section{References}

[BCJ08] Ali Bagherzandi, Jung-Hee Cheon, and Stanislaw Jarecki. Multisignatures secure under the discrete logarithm assumption and a generalized forking lemma. In Proceedings of the 15th ACM conference on Computer and communications security, CCS '08, pages 449-458, New York, NY, USA, 2008. ACM. (Cited on page 3.) 
[BF01] Dan Boneh and Matt Franklin. Identity-based encryption from the weil pairing. In Joe Kilian, editor, Advances in Cryptology - CRYPTO 2001, volume 2139 of Lecture Notes in Computer Science, pages 213-229. Springer Berlin / Heidelberg, 2001. (Cited on page 10.)

[BN06] Mihir Bellare and Gregory Neven. Multi-signatures in the plain public-key model and a general forking lemma. In Proceedings of the 13th ACM conference on Computer and communications security, CCS '06, pages 390-399, New York, NY, USA, 2006. ACM. (Cited on pages 3, 4, 5, 8, 14, 15 and 26.)

[BNN04] Mihir Bellare, Chanathip Namprempre, and Gregory Neven. Security proofs for identity-based identification and signature schemes. In Christian Cachin and Jan Camenisch, editors, Advances in Cryptology - EUROCRYPT 2004, volume 3027 of Lecture Notes in Computer Science, pages 268-286. Springer Berlin / Heidelberg, 2004. (Cited on page 20.)

[BPW12] Alexandra Boldyreva, Adriana Palacio, and Bogdan Warinschi. Secure proxy signature schemes for delegation of signing rights. Journal of Cryptology, 25:57-115, 2012. (Cited on pages 3, 4, 5, 6, 7, 9, 10, 13, 16, 17, 19, 20, 27, 28 and 36.)

[BR93] Mihir Bellare and Phillip Rogaway. Random oracles are practical: a paradigm for designing efficient protocols. In Proceedings of the 1st ACM conference on Computer and communications security, CCS '93, pages 62-73, New York, NY, USA, 1993. ACM. (Cited on page 3.)

[CKK13] Sanjit Chatterjee, Chethan Kamath, and Vikas Kumar. Galindo-Garcia identitybased signature revisited. In Taekyoung Kwon, Mun-Kyu Lee, and Daesung Kwon, editors, Information Security and Cryptology - ICISC 2012, volume 7839 of Lecture Notes in Computer Science, pages 456-471. Springer Berlin / Heidelberg, 2013. Full version available in Cryptology ePrint Archive, Report 2012/646, http://eprint.iacr.org/2012/646. (Cited on pages 3, 4, 21, 22 and 39.)

[CMW12] Sherman S. M. Chow, Changshe Ma, and Jian Weng. Zero-knowledge argument for simultaneous discrete logarithms. Algorithmica, 64(2):246-266, 2012. (Cited on pages $3,4,5,6,7,9,10,17$ and 22 .)

[CP93] David Chaum and Torben P. Pedersen. Wallet databases with observers. In Proceedings of the 12th Annual International Cryptology Conference on Advances in Cryptology, CRYPTO '92, pages 89-105, London, UK, UK, 1993. Springer-Verlag. (Cited on page 38.)

[ElG85] Taher ElGamal. A public key cryptosystem and a signature scheme based on discrete logarithms. In GeorgeRobert Blakley and David Chaum, editors, Advances in Cryptology, volume 196 of Lecture Notes in Computer Science, pages 10-18. Springer Berlin Heidelberg, 1985. (Cited on page 3.)

[FS87] Amos Fiat and Adi Shamir. How to prove yourself: Practical solutions to identification and signature problems. In Andrew Odlyzko, editor, Advances in Cryptology - CRYPTO' 86, volume 263 of Lecture Notes in Computer Science, pages 186-194. Springer Berlin / Heidelberg, 1987. (Cited on pages 3, 19 and 21.)

[GG09] David Galindo and Flavio Garcia. A Schnorr-like lightweight identity-based signature scheme. In Bart Preneel, editor, Progress in Cryptology - AFRICACRYPT 2009, volume 5580 of Lecture Notes in Computer Science, pages 135-148. Springer Berlin / Heidelberg, 2009. (Cited on pages 3, 4, 5, 6, 9, 10, 17 and 20.) 
[Oka93] Tatsuaki Okamoto. Provably secure and practical identification schemes and corresponding signature schemes. In ErnestF. Brickell, editor, Advances in Cryptology - CRYPTO' 92, volume 740 of Lecture Notes in Computer Science, pages 31-53. Springer Berlin Heidelberg, 1993. (Cited on page 3.)

[PS00] David Pointcheval and Jacques Stern. Security arguments for digital signatures and blind signatures. Journal of Cryptology, 13:361-396, 2000. (Cited on page 3.)

[Sch91] Claus-Peter Schnorr. Efficient signature generation by smart cards. Journal of Cryptology, 4:161-174, 1991. 10.1007/BF00196725. (Cited on page 3.)

[Seu12] Yannick Seurin. On the exact security of Schnorr-type signatures in the random oracle model. In David Pointcheval and Thomas Johansson, editors, Advances in Cryptology - EUROCRYPT 2012, volume 7237 of Lecture Notes in Computer Science, pages 554-571. Springer Berlin / Heidelberg, 2012. (Cited on page 4.)

$\left[Y_{A D V}{ }^{+} 12\right]$ Sidi-Mohamed Yousfi-Alaoui, Özgür Dagdelen, Pascal Véron, David Galindo, and Pierre-Louis Cayrel. Extended security arguments for signature schemes. In Aikaterini Mitrokotsa and Serge Vaudenay, editors, Progress in Cryptology AFRICACRYPT 2012, volume 7374 of Lecture Notes in Computer Science, pages 1934. Springer Berlin Heidelberg, 2012. (Cited on page 5.)

[YZ13] Andrew Chi-Chih Yao and Yunlei Zhao. Online/offline signatures for low-power devices. IEEE Transactions on Information Forensics and Security, 8(2):283-294, 2013. (Cited on page 5.)

\section{A General Forking}

We reproduce the GF Algorithm from [BN06] followed by the statement of the GF lemma. We use slightly different notations to maintain uniformity.

Forking Algorithm. Fix $q \in \mathbb{Z}^{+}$and a set $\mathbb{S}$ such that $|\mathbb{S}| \geq 2$. Let $\mathcal{Y}$ be a randomised algorithm that on input a string $x$ and elements $s_{1}, \ldots, s_{q} \in \mathbb{S}$ returns a pair $(I, \sigma)$ consisting of an integer $0 \leq I \leq q$ and a string $\sigma$. The forking algorithm $\mathcal{F}_{\mathcal{Y}}$ associated to $\mathcal{Y}$ is defined as Algorithm 3 below.

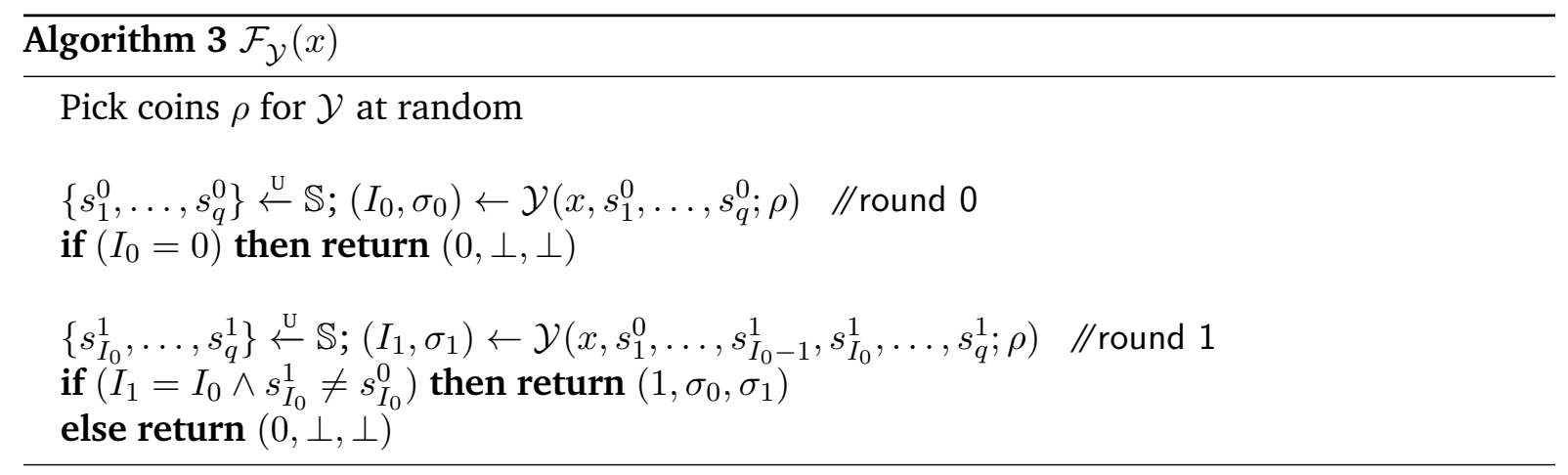

Lemma 7 (General Forking Lemma [BN06]). Let $\mathcal{G}_{I}$ be a randomised algorithm that takes no input and returns a string. Let

$$
\begin{gathered}
\text { gfrk }:=\operatorname{Pr}\left[(b=1) \mid x \stackrel{\$}{\leftarrow} \mathcal{G}_{I} ;(b, \sigma, \sigma) \stackrel{\$}{\leftarrow} \mathcal{F}_{\mathcal{Y}}(x)\right] \text { and } \\
\text { acc }:=\operatorname{Pr}\left[I \geq 1 \mid x \stackrel{\$}{\leftarrow} \mathcal{G}_{I} ;\left\{s_{1}, \ldots, s_{q}\right\} \stackrel{\cup}{\leftarrow} \mathbb{S} ;(I, \sigma) \stackrel{\$}{\leftarrow} \mathcal{Y}\left(x, s_{1}, \ldots, s_{q}\right)\right],
\end{gathered}
$$


then

$$
\operatorname{gfrk} \geq \operatorname{acc} \cdot\left(\frac{\operatorname{acc}}{q}-\frac{1}{|\mathbb{S}|}\right)
$$

\section{B (Original) Multiple-Forking Algorithm}

We describe the original Multiple-Forking Algorithm [BPW12] with some notational changes. The success condition and the lemma that governs the success probability follows the algorithm.

The Multiple-Forking Algorithm Fix $q \in \mathbb{Z}^{+}$and a set $\mathbb{S}$ such that $|\mathbb{S}| \geq 2$. Let $\mathcal{Y}$ be a randomised algorithm that on input a string $x$ and elements $s_{1}, \ldots, s_{q} \in \mathbb{S}$ returns a triple $(I, J, \sigma)$ consisting of two integers $0 \leq J<I \leq q$ and a string $\sigma$. Let $n \geq 1$ be an odd integer. The MF Algorithm $\mathcal{M}_{\mathcal{Y}, n}$ associated to $\mathcal{Y}$ and $n$ is defined as Algorithm 4 below.

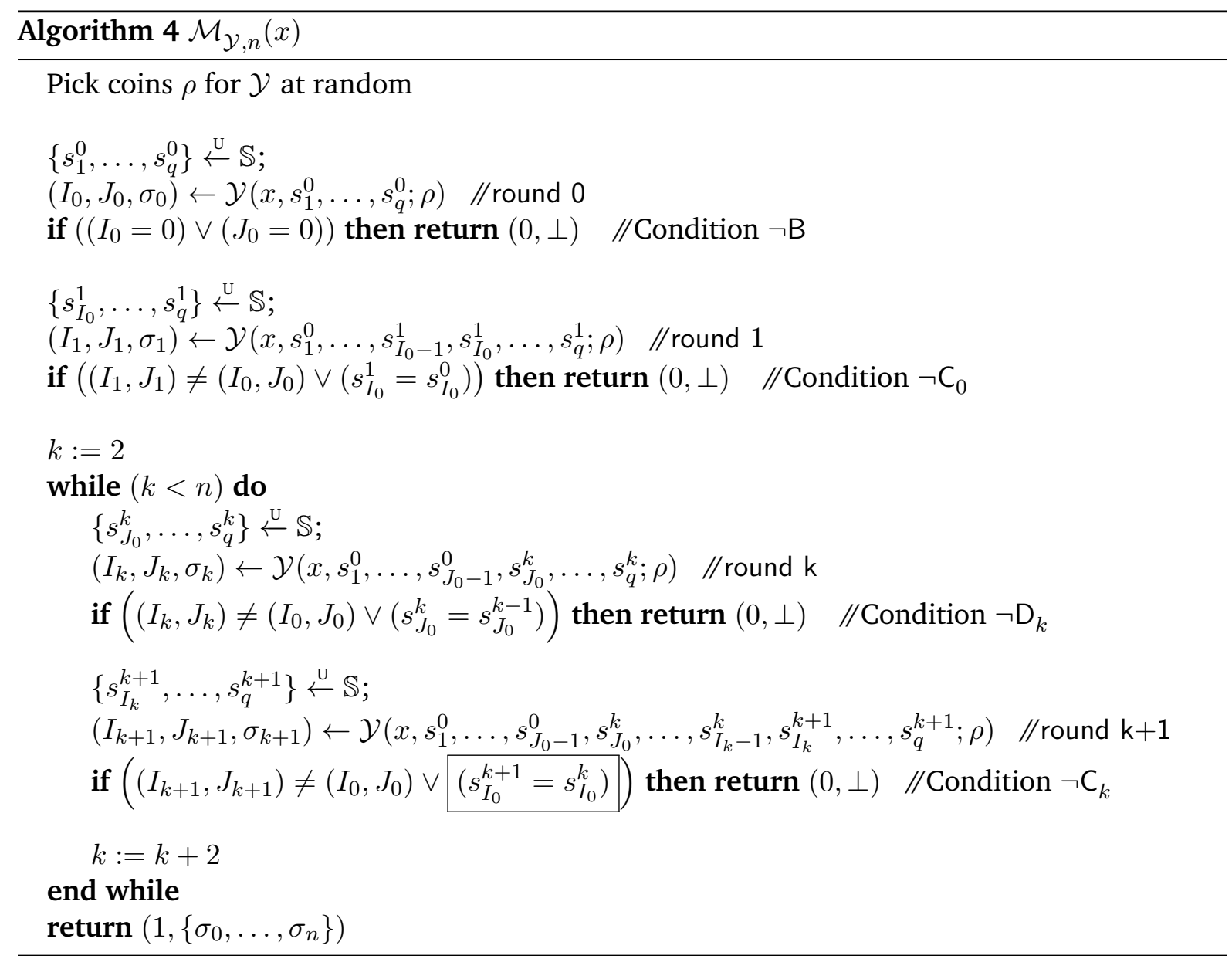

The success condition. The success of the MF Algorithm is determined by the set of conditions $\mathbb{A}_{0}:=\left\{\mathrm{B}, \mathrm{C}_{0}, \ldots, \mathrm{C}_{n-1}, \mathrm{C}_{2}, \ldots, \mathrm{D}_{n-1}\right\}$ where

$$
\begin{aligned}
\mathrm{B} & :\left(I_{0} \geq 1\right) \wedge\left(J_{0} \geq 1\right) \\
\mathrm{C}_{k} & :\left(I_{k+1}, J_{k+1}\right)=\left(I_{k}, J_{k}\right) \wedge\left(s_{I_{k}}^{k+1} \neq s_{I_{k}}^{k}\right) \quad(\text { for } k=0,2, \ldots, n-1) \\
\mathrm{D}_{k} & :\left(I_{k}, J_{k}\right)=\left(I_{0}, J_{0}\right) \wedge\left(s_{J_{0}}^{k} \neq s_{J_{0}}^{k-1}\right) \quad(\text { for } k=2,4, \ldots, n-1)
\end{aligned}
$$

To be precise, the MF Algorithm is successful in the event $E$ that all of the conditions in $\mathbb{A}_{0}$ are satisfied, i.e.,

$$
E: B \wedge\left(C_{0} \wedge C_{2} \wedge \cdots \wedge C_{n-1}\right) \wedge\left(D_{2} \wedge D_{4} \wedge \cdots \wedge D_{n-1}\right)
$$


The probability of this event, which is denoted by $m f r k$, is bounded by the MF lemma given below.

Lemma 8 ((Original) Multiple-Forking Lemma [BPW12]). Let $\mathcal{G}_{I}$ be a randomised algorithm that takes no input and returns a string. Let

$$
\begin{gathered}
\text { mfrk }:=\operatorname{Pr}\left[(b=1) \mid x \stackrel{\$}{\leftarrow} \mathcal{G}_{I} ;\left(b,\left\{\sigma_{0}, \ldots, \sigma_{n}\right\}\right) \stackrel{\$}{\leftarrow} \mathcal{M}_{\mathcal{Y}, n}(x)\right] \text { and } \\
\text { acc }:=\operatorname{Pr}\left[(I \geq 1) \wedge(J \geq 1) \mid x \stackrel{\$}{\leftarrow} \mathcal{G}_{I} ;\left\{s_{1}, \ldots, s_{q}\right\} \stackrel{\cup}{\leftarrow} \mathbb{S} ;(I, J, \sigma) \stackrel{\$}{\leftarrow} \mathcal{Y}\left(x, s_{1}, \ldots, s_{q}\right)\right]
\end{gathered}
$$

then

$$
m f r k \geq a c c \cdot\left(\frac{a c c^{n}}{q^{2 n}}-\frac{n}{|\mathbb{S}|}\right)
$$

\section{Harnessing (In)Dependence}

\section{C.1 Detailed Steps for Lemma 2}

\section{Intermediate steps for (13)}

$$
\begin{aligned}
& \operatorname{frk}_{c}(x) \\
& =\operatorname{Pr}\left[\left(I_{1}=I_{0}\right) \wedge\left(J_{1}=J_{0}\right) \wedge\left(1 \leq J_{0}<I_{0} \leq q\right)\right] \\
& =\operatorname{Pr}\left[\left(J_{1}=J_{0}\right) \mid\left(I_{1}=I_{0}\right) \wedge\left(1 \leq J_{0}<I_{0} \leq q\right)\right] \operatorname{Pr}\left[\left(I_{1}=I_{0}\right) \wedge\left(1 \leq J_{0}<I_{0} \leq q\right)\right] \\
& =(1-\eta) \operatorname{Pr}\left[\left(I_{1}=I_{0}\right) \wedge\left(1 \leq J_{0}<I_{0} \leq q\right)\right] \\
& =(1-\eta) \sum_{i=2}^{q} \operatorname{Pr}\left[\left(I_{1}=i\right) \wedge\left(1 \leq J_{1}<i\right) \mid\left(I_{0}=i\right) \wedge\left(1 \leq J_{0}<i\right)\right] \cdot \operatorname{Pr}\left[\left(I_{0}=i\right) \wedge\left(1 \leq J_{0}<i\right)\right] \\
& =(1-\eta) \sum_{i=2}^{q} \sum_{\rho, \mathrm{S}_{(i-)}} \frac{1}{|\mathbb{R}||\mathbb{S}|^{j-1}} Y_{i}^{2}\left(\rho, \mathrm{S}_{(i-)}\right) \\
& \geq \frac{(1-\eta)}{q}\left(\sum_{i=2}^{q} \operatorname{Ex}\left[Y_{i}^{2}\right] \geq \sum_{i=2}^{q} \operatorname{Ex}\left[Y_{i}\right]^{2} \quad\right. \text { (by Jensen's inequality) } \\
& =\frac{(1-\eta)}{q} \text { acc }(x)^{2} \quad \text { (by definition of } Y_{i} \text { and acc(x)) }
\end{aligned}
$$




\section{Intermediate steps for (17)}

$$
\begin{aligned}
& m f r k_{3, c}(x) \\
= & \operatorname{Pr}\left[\left(1 \leq J_{0}<I_{0} \leq q\right) \wedge\left(I_{1}=I_{0}\right) \wedge\left(s_{I_{0}}^{1} \neq s_{I_{0}}^{0}\right) \wedge\right. \\
& \left.\quad\left(\wedge_{k=2,4, \ldots, n-1}\left(I_{k+1}=I_{k}\right) \wedge\left(s_{I_{k}}^{k+1} \neq s_{I_{k}}^{k}\right) \wedge\left(J_{k}=J_{0}\right) \wedge\left(J_{k}<I_{k} \leq q\right)\right)\right] \\
= & \operatorname{Pr}\left[\left(1 \leq J_{0}<I_{0} \leq q\right) \wedge\left(I_{1}=I_{0}\right) \wedge\left(J_{1}=J_{0}\right) \wedge\left(s_{I_{0}}^{1} \neq s_{I_{0}}^{0}\right) \wedge\right. \\
& \left.\quad\left(\wedge_{k=2,4, \ldots, n-1}\left(I_{k+1}=I_{k}\right) \wedge\left(s_{I_{k}}^{k+1} \neq s_{I_{k}}^{k}\right) \wedge\left(J_{k+1}=J_{k}=J_{0}\right) \wedge\left(J_{k}<I_{k} \leq q\right)\right)\right] \quad(\text { since } J \prec I) \\
= & \sum_{j=1}^{q-1} \operatorname{Pr}\left[\wedge_{k=0,2, \ldots, n-1}\left(\left(J_{k+1}=J_{k}=j\right) \wedge\left(I_{k+1}=I_{k}\right) \wedge\left(j<I_{k} \leq q\right) \wedge\left(s_{I_{k}}^{k+1} \neq s_{I_{k}}^{k}\right)\right)\right] \\
= & \sum_{j=1}^{q-1} \sum_{\rho, \mathrm{S}_{(j-)}} \frac{\operatorname{Pr}\left[\wedge_{k=0,2, \ldots, n-1}\left(J_{k+1}=J_{k}=j\right) \wedge\left(I_{k+1}=I_{k}\right) \wedge\left(j<I_{k} \leq q\right) \wedge\left(s_{I_{k}}^{k+1} \neq s_{I_{k}}^{k}\right)\right]}{|\mathbb{R}||\mathbb{S}|^{j-1}} \\
= & \sum_{j=1}^{q-1} \sum_{\rho, \mathrm{S}_{(j-)}} \frac{\prod_{k=0,2}^{n-1} \operatorname{Pr}\left[\left(J_{k+1}=J_{k}=j\right) \wedge\left(I_{k+1}=I_{k}\right) \wedge\left(j<I_{k} \leq q\right) \wedge\left(s_{I_{k}}^{k+1} \neq s_{I_{k}}^{k}\right) \mid \bigwedge_{l=0,2}^{k-2} \mathrm{G}_{\ell}\right]}{|\mathbb{R}||\mathbb{S}|^{j-1}}
\end{aligned}
$$

In the above expression, $\mathrm{G}_{\ell}$ denotes the event

$$
\left(\left(J_{\ell+1}=J_{\ell}=j\right) \wedge\left(I_{\ell+1}, J_{\ell+1}\right)=\left(I_{\ell}, J_{\ell}\right) \wedge\left(j<I_{\ell} \leq q\right) \wedge\left(s_{I_{\ell}}^{\ell+1} \neq s_{I_{\ell}}^{\ell}\right) .\right.
$$

Using the random variable $Z_{j}$, the equation (17) can be rewritten as

$$
\begin{aligned}
& m f r k_{3, c}(x) \\
& =\sum_{j=1}^{q-1} \sum_{\rho, \mathrm{S}} \frac{\prod_{(j-)}^{n-1} Z_{j=0,2}\left(\rho, \mathrm{S}_{(j-)}\right)}{|\mathbb{R}||\mathbb{S}|^{j-1}}=\sum_{j=1}^{q-1}\left(\sum_{\rho, \mathrm{S}_{(j-)}} \frac{Z_{j}^{(n+1) / 2}\left(\rho, \mathrm{S}_{(j-)}\right)}{|\mathbb{R}||\mathbb{S}|^{j-1}}\right) \\
& =\sum_{j=1}^{q-1} \operatorname{Ex}\left[Z_{j}^{(n+1) / 2}\right] \geq \sum_{j=1}^{q-1} \operatorname{Ex}\left[Z_{j}\right]^{(n+1) / 2} \quad \text { (by Jensen's inequality) } \\
& \geq \frac{1}{q^{(n-1) / 2}}\left(\sum_{j=1}^{q-1} \operatorname{Ex}\left[Z_{j}\right]\right)^{(n+1) / 2} \quad \text { (by Jensen's and Hölder's inequality) }
\end{aligned}
$$

\section{Intermediate steps for (18)}

$$
\begin{aligned}
& \text { frk }_{3, s}(x)= \\
& =\operatorname{Pr}\left[\left(1 \leq J_{0}<I_{0} \leq q\right) \wedge\left(I_{1}=I_{0}\right) \wedge\left(s_{I_{0}}^{1} \neq s_{I_{0}}^{0}\right)\right] \cdot \operatorname{Pr}\left[\left(\vee_{k=2,4, \ldots, n-1} \neg \mathrm{D}_{k, s}\right)\right] \\
& =\frac{(n-1)(n+1)}{8|\mathbb{S}|} \cdot \operatorname{Pr}\left[\left(1 \leq J_{0}<I_{0} \leq q\right) \wedge\left(I_{1}=I_{0}\right) \wedge\left(J_{1}=J_{0}\right) \wedge\left(s_{I_{0}}^{1} \neq s_{I_{0}}^{0}\right)\right] \\
& =\frac{(n-1)(n+1)}{8|\mathbb{S}|}\left(\sum_{j=1}^{q-1}\left(J_{1}=J_{0}=j\right) \wedge\left(I_{1}=I_{0}\right) \wedge\left(j<I_{0} \leq q\right) \wedge\left(s_{I_{0}}^{1} \neq s_{I_{0}}^{0}\right)\right) \\
& =\frac{(n-1)(n+1)}{8|\mathbb{S}|}\left(\sum_{j=1}^{q-1} \operatorname{Ex}\left[Z_{j}\right]\right)
\end{aligned}
$$




\section{C.2 Multiple-Forking with Index Independence}

Lemma 9 (Multiple-Forking Lemma with Index Independence). Let $\mathcal{G}_{I}$ be a randomised algorithm that takes no input and returns a string. Let

$$
\begin{gathered}
m_{\text {rrk }}:=\operatorname{Pr}\left[(b=1) \mid x \stackrel{\$}{\leftarrow} \mathcal{G}_{I} ;\left(b,\left\{\sigma_{0}, \ldots, \sigma_{n}\right\}\right) \stackrel{\$}{\leftarrow} \mathcal{N}_{\mathbb{A}_{1}, \mathcal{Y}, n}(x)\right] \text { and } \\
\text { acc }:=\operatorname{Pr}\left[(I \geq 1) \wedge(J \geq 1) \mid x \stackrel{\$}{\leftarrow} \mathcal{G}_{I} ;\left\{s_{1}, \ldots, s_{q}\right\} \stackrel{\cup}{\leftarrow} \mathbb{S} ;(I, J, \sigma) \stackrel{\$}{\leftarrow}\left(x, s_{1}, \ldots, s_{q}\right)\right]
\end{gathered}
$$

then

$$
m f r k_{1} \geq a c c \cdot\left(\frac{a c c^{n}}{q^{(3 n+1) / 2}}-\frac{(n+1)(n+3)}{8|\mathbb{S}|}\right) .
$$

Proof. The analysis, especially its logical flow, is quite similar to the original analysis. We stick to the conventions adopted in $\S 3.1$. For a fixed string $x$, let

$$
\begin{gathered}
m \operatorname{mrk}_{1}(x):=\operatorname{Pr}\left[(\mathrm{b}=1) \mid\left(\mathrm{b},\left\{\sigma_{0}, \ldots, \sigma_{n}\right\}\right) \stackrel{\$}{\leftarrow} \mathcal{N}_{\mathbb{A}_{1}, \mathcal{Y}, n}(x)\right] \text { and } \\
\operatorname{acc}(x):=\operatorname{Pr}\left[(I \geq 1) \wedge(J \geq 1) \mid\left\{s_{1}, \ldots, s_{q}\right\} \stackrel{\amalg}{\leftarrow} \mathbb{S} ;(I, J, \sigma) \stackrel{\$}{\leftarrow} \mathcal{Y}\left(x, s_{1}, \ldots, s_{q}\right)\right]
\end{gathered}
$$

Let $\mathbb{A}_{1}:=\left\{B, C_{0}, C_{2}, \ldots, C_{n-1}, D_{2}, D_{4}, \ldots, D_{n-1}\right\}$. For ease of notation, we further break the event $\mathrm{C}_{k}$ (resp. $\mathrm{D}_{k}$ ) into two subevents $\mathrm{C}_{k, c}$ and $\mathrm{C}_{k, s}$ (resp. $\mathrm{D}_{k, c}$ and $\mathrm{D}_{k, s}$ ) as follows:

$$
\begin{aligned}
\mathrm{C}_{k, c}:\left(I_{k+1}, J_{k+1}\right)=\left(I_{k}, J_{k}\right) \wedge\left(I_{k} \geq 1\right) & \mathrm{C}_{k, s}:\left(s_{I_{k}}^{k+1} \neq s_{I_{k}}^{k}\right) \\
\mathrm{D}_{k, c}:\left(J_{k}=J_{0}\right) & \mathrm{D}_{k, s}:\left(\wedge_{\ell:=0,2, \ldots, k-2} s_{J_{0}}^{k} \neq s_{J_{0}}^{\ell}\right)
\end{aligned}
$$

The GMF Algorithm is successful in the event $E: B \wedge\left(C_{0} \wedge C_{2} \wedge \cdots \wedge C_{n-1}\right) \wedge\left(D_{2} \wedge D_{4} \wedge \cdots \wedge D_{n-1}\right)$. In other words, with the probabilities calculated over the randomness of the GMF Algorithm, it follows that $m f r k_{1}(x)=\operatorname{Pr}[E]$. The first step in calculating the probability is to separate the "core" subevents out of the event E. This is accomplished as follows.

$$
\begin{aligned}
\operatorname{Pr}[\mathrm{E}]= & \operatorname{Pr}\left[\mathrm{B} \wedge\left(\wedge_{k=0,2, \ldots, n-1} \mathrm{C}_{k}\right) \wedge\left(\wedge_{k=2,4, \ldots, n-1} \mathrm{D}_{k}\right)\right] \\
= & \operatorname{Pr}\left[\mathrm{B} \wedge\left(\wedge_{k=0,2, \ldots, n-1} \mathrm{C}_{k, c} \wedge \mathrm{C}_{k, s}\right) \wedge\left(\wedge_{k=2,4, \ldots, n-1} \mathrm{D}_{k, c} \wedge \mathrm{D}_{k, s}\right)\right] \text { (using (33)) } \\
\geq & \operatorname{Pr}\left[\mathrm{B} \wedge\left(\wedge_{k=0,2, \ldots, n-1} \mathrm{C}_{k, c}\right) \wedge\left(\wedge_{k=2,4, \ldots, n-1} \mathrm{D}_{k, c}\right)\right]- \\
& \operatorname{Pr}\left[\mathrm{B} \wedge\left(\left(\vee_{k=0,2, \ldots, n-1} \mathrm{C}_{k, s}\right) \vee\left(\vee_{k=2,4, \ldots, n-1} \mathrm{D}_{k, s}\right)\right)\right]
\end{aligned}
$$

It can be shown that the second part of (34) equals

$$
\frac{(n+1)(n+3) \cdot \operatorname{acc}(x)}{8|\mathbb{S}|}
$$

by following the analysis in (18). As for the first part, it constitutes the "core" event for the analysis, and is denoted by $m f r k_{1, c}(x)$. The event corresponding to $m f r k_{1, c}(x)$ is closely related to the event given in (6). The next step is to show that

$$
m f r k_{1, c}(x) \geq \frac{\operatorname{acc}(x)^{n+1}}{q^{(3 n+1) / 2}} .
$$


The intermediate steps to achieving it are as follows:

$$
\begin{aligned}
\operatorname{mfrk}_{1, c}(x) & =\operatorname{Pr}\left[\mathrm{B} \wedge\left(\wedge_{k=0,2, \ldots, n-1} \mathrm{C}_{k, c}\right) \wedge\left(\wedge_{k=2,4, \ldots, n-1} \mathrm{D}_{k, c}\right)\right] \\
& =\operatorname{Pr}\left[\left(I_{0} \geq 1\right) \wedge\left(J_{0} \geq 1\right) \wedge \bigwedge_{k=0,2}^{n-1}\left(\left(I_{k+1}, J_{k+1}\right)=\left(I_{k}, J_{k}\right) \wedge\left(I_{k} \geq 1\right)\right) \wedge \bigwedge_{k=2,4}^{n-1}\left(J_{k}=J_{0}\right)\right] \\
& =\sum_{j=1}^{q} \operatorname{Pr}\left[\left(I_{0} \geq 1\right) \wedge\left(J_{0}=j\right) \wedge \bigwedge_{k=0,2}^{n-1}\left(\left(I_{k+1}, J_{k+1}\right)=\left(I_{k}, J_{k}\right) \wedge\left(I_{k} \geq 1\right)\right) \wedge \bigwedge_{k=2,4}^{n-1}\left(J_{k}=j\right)\right] \\
& =\sum_{j=1}^{q} \sum_{\rho, \mathrm{S}_{(j-)}} \frac{\prod_{k=0,2}^{n-1} \operatorname{Pr}\left[\left(I_{k+1}, J_{k+1}\right)=\left(I_{k}, J_{k}\right) \wedge\left(J_{k}=j\right) \wedge\left(I_{k} \geq 1\right) \mid \bigwedge_{l=2,4}^{k-2} \mathrm{G}_{\ell}\right]}{|\mathbb{R}||\mathbb{S}|^{j-1}}
\end{aligned}
$$

In the above expression, $\mathrm{G}_{\ell}$ denotes the event $\left(I_{\ell+1}, J_{\ell+1}\right)=\left(I_{\ell}, J_{\ell}\right) \wedge\left(J_{\ell}=j\right) \wedge\left(I_{\ell} \geq 1\right)$. Let's focus on the probability part of (36).

$$
\begin{aligned}
& \operatorname{Pr}\left[\left(I_{k+1}, J_{k+1}\right)=\left(I_{k}, J_{k}\right) \wedge\left(J_{k}=j\right) \wedge\left(I_{k} \geq 1\right)\right] \\
& =\sum_{i=1}^{q} \operatorname{Pr}\left[\left(I_{k+1}, J_{k+1}\right)=\left(I_{k}, J_{k}\right)=(i, j)\right] \\
& =\sum_{i=1}^{q} \sum_{\mathbf{S}_{(j i)}} \frac{\operatorname{Pr}\left[\left(I_{k+1}, J_{k+1}\right)=\left(I_{k}, J_{k}\right)=(i, j)\right]}{|\mathbb{S}|^{i-j}} \\
& =\sum_{i=1}^{q} \sum_{\mathbf{S}_{(j i)}} \frac{\operatorname{Pr}\left[\left(I_{k+1}, J_{k+1}\right)=(i, j)\right] \cdot \operatorname{Pr}\left[\left(I_{k}, J_{k}\right)=(i, j)\right]}{|\mathbb{S}|^{i-j}}
\end{aligned}
$$

At this point, we define a random variable $Y_{i, j, \rho, \mathrm{S}_{(j-)}}$ which captures a single invocation of the wrapper $\mathcal{Y}$, but with the internal randomness $\rho$ and the external randomness $\mathrm{S}_{(j-)}$ being fixed. Let $Y_{i, j, \rho, \mathrm{S}_{(j-)}}: \mathbb{S}^{i-j} \mapsto[0,1]$, for each $i, j \in\{1, \ldots, q\}$, be defined by setting

$$
Y_{i, j, \rho, \mathrm{S}_{(j-)}}\left(\mathrm{S}_{(j i)}\right):=\operatorname{Pr}\left[(I, J)=(i, j) \mid \mathrm{S}_{(i+)} \stackrel{\$}{\leftarrow} \mathbb{S}^{q-i+1} ;(I, J, \sigma) \leftarrow \mathcal{Y}\left(x, \mathrm{~S}_{(j-)}, \mathrm{S}_{(j i)}, \mathrm{S}_{(i+)} ; \rho\right)\right]
$$

Using the random variable $Y$, the equation (37) can be rewritten as

$$
\begin{aligned}
& \sum_{i=1}^{q} \sum_{\mathrm{S}_{(j i)}} \frac{Y_{i, j, \rho, \mathrm{S}_{(j-)}}^{2}\left(\mathrm{~S}_{(j i)}\right)}{\mid \mathbb{S}^{i-j}} \\
& =\sum_{i=1}^{q} \operatorname{Ex}\left[Y_{i, j, \rho, \mathbf{S}_{(j-)}}^{2}\right] \geq \sum_{i=1}^{q} \operatorname{Ex}\left[Y_{i, j, \rho, \mathrm{S}_{(j-)}}\right]^{2} \quad \text { (by Jensen's inequality) } \\
& \geq \frac{1}{q}\left(\sum_{i=1}^{q} \operatorname{Ex}\left[Y_{i, j, \rho, \mathbf{S}_{(j-)}}\right]\right)^{2} \quad \text { (by Hölder's inequality) }
\end{aligned}
$$

Next, we define a random variable $Z_{j}$ which captures one invocation of the logical wrapper $\mathcal{Z}$. Let $Z_{j}: \mathbb{R} \times \mathbb{S}^{j-1} \mapsto[0,1]$, for each $j \in\{1, \ldots, q\}$, be defined by setting

$$
Z_{j}\left(\rho, \mathrm{S}_{(j-)}\right):=\sum_{i=1}^{q} \operatorname{Ex}\left[Y_{i, j, \rho, \mathrm{S}_{(j-)}}\right] .
$$

Hence, on representing (38) in terms of the random variable $Z_{j}$, we get

$$
\operatorname{Pr}\left[\left(I_{k+1}, J_{k+1}\right)=\left(I_{k}, J_{k}\right) \wedge\left(J_{k}=j\right) \wedge\left(I_{k} \geq 1\right)\right] \geq Z_{j}\left(\rho, \mathrm{S}_{(j-)}\right)^{2} / q
$$


Substituting the above expression, further, in (36) yields

$$
\begin{aligned}
& \sum_{j=1}^{q} \sum_{\rho, \mathrm{S}_{(j-)}} \frac{\prod_{k=0,2}^{n-1} \operatorname{Pr}\left[\left(I_{k+1}, J_{k+1}\right)=\left(I_{k}, J_{k}\right) \wedge\left(J_{k}=j\right) \wedge\left(I_{k} \geq 1\right)\right]}{|\mathbb{R}||\mathbb{S}|^{j-1}} \\
& \geq \sum_{j=1}^{q} \sum_{\rho, \mathrm{S}_{(j-)}} \frac{\left(Z_{j}\left(\rho, \mathrm{S}_{(j-)}\right)^{2} / q\right)^{(n+1) / 2}}{|\mathbb{R}||\mathbb{S}|^{j-1}} \\
& =\frac{1}{q^{(n+1) / 2}} \sum_{j=1}^{q} \sum_{\rho, \mathbf{S}_{(j-)}} \frac{Z_{j}\left(\rho, \mathrm{S}_{(j-)}\right)^{n+1}}{|\mathbb{R}||\mathbb{S}|^{j-1}} \\
& =\frac{1}{q^{(n+1) / 2}} \sum_{j=1}^{q} \operatorname{Ex}\left[Z_{j}^{n+1}\right] \geq \frac{1}{q^{(n+1) / 2}} \sum_{j=1}^{q} \operatorname{Ex}\left[Z_{j}\right]^{n+1} \quad \text { (by Jensen's inequality) } \\
& \geq \frac{1}{q^{(n+1) / 2} \cdot q^{n}}\left(\sum_{j=1}^{q} \operatorname{Ex}\left[Z_{j}\right]\right)^{n+1} \quad \text { (by Hölder's inequality) } \\
& =\frac{1}{q^{(3 n+1) / 2}} \operatorname{acc}(x)^{n+1} .
\end{aligned}
$$

That completes the analysis of the "core" event and establishes our initial claim in (35). On combining the two parts of the equation (34), we get

$$
\begin{aligned}
m f r k_{1}(x) & \geq \frac{\operatorname{acc}(x)^{n+1}}{q^{(3 n+1) / 2}}-\frac{(n+1)(n+3) \cdot \operatorname{acc}(x)}{8|\mathbb{S}|} \\
& =\operatorname{acc}(x)\left(\frac{\operatorname{acc}(x)^{n}}{q^{(3 n+1) / 2}}-\frac{(n+1)(n+3)}{8|\mathbb{S}|}\right) .
\end{aligned}
$$

With expectation taken over $x \stackrel{\$}{\leftarrow} \mathcal{G}_{I}$,

$$
m f r k_{1} \geq \operatorname{acc}\left(\frac{a c c^{n}}{q^{(3 n+1) / 2}}-\frac{(n+1)(n+3)}{8|\mathbb{S}|}\right) .
$$

hence, proving the lemma. We conclude with the comment that on assuming $|\mathbb{S}| \gg 1$, one gets $m f r k_{2} \approx a c c^{n+1} / q^{(3 n 1) / 2}$.

\section{C.3 Multiple-Forking with Index Dependence}

Lemma 10 (Multiple-Forking Lemma with Index Dependence). Let $\mathcal{G}_{I}$ be a randomised algorithm that takes no input and returns a string. Let

$$
\begin{gathered}
m f r k:=\operatorname{Pr}\left[(b=1) \mid x \stackrel{\$}{\leftarrow} \mathcal{G}_{I} ;\left(b,\left\{\sigma_{0}, \ldots, \sigma_{n}\right\}\right) \stackrel{\$}{\leftarrow} \mathcal{N}_{\mathbb{A}_{0}, \mathcal{Y}, n}(x)\right] \text { and } \\
\text { acc }:=\operatorname{Pr}\left[(1 \leq J<I \leq q) \mid x \stackrel{\$}{\leftarrow} \mathcal{G}_{I} ;\left\{s_{1}, \ldots, s_{q}\right\} \stackrel{\amalg}{\leftarrow} \mathbb{S} ;(I, J, \sigma) \stackrel{\$}{\leftarrow} \mathcal{Y}\left(x, s_{1}, \ldots, s_{q}\right)\right]
\end{gathered}
$$

On the assumption that $J$ is $\eta$-dependent on $I$,

$$
m f r k \geq f r k\left(\frac{f r k^{(n-1) / 2}}{q^{n}}-\frac{(n-1)(n+1)}{8|\mathbb{S}|}\right) \text { where frk } \geq \operatorname{acc}\left(\frac{\operatorname{acc}}{q}-\frac{1}{|\mathbb{S}|}\right)
$$


Proof. For a fixed string $x$, let

$$
\begin{gathered}
m f r k(x):=\operatorname{Pr}\left[(\mathrm{b}=1) \mid\left(\mathrm{b},\left\{\sigma_{0}, \ldots, \sigma_{n}\right\}\right) \stackrel{\$}{\leftarrow} \mathcal{N}_{\mathbb{A}_{0}, \mathcal{Y},(x)}\right. \text { and } \\
\operatorname{acc}(x):=\operatorname{Pr}\left[(1 \leq J<I \leq q) \mid\left\{s_{1}, \ldots, s_{q}\right\} \stackrel{\mathrm{U}}{\leftarrow} \mathbb{S} ;(I, J, \sigma) \stackrel{\$}{\leftarrow} \mathcal{Y}\left(x, s_{1}, \ldots, s_{q}\right)\right] .
\end{gathered}
$$

Let $\mathbb{A}_{1}:=\left\{B, C_{0}, C_{2}, \ldots, C_{n-1}, D_{2}, D_{4}, \ldots, D_{n-1}\right\}$. For ease of notation, we further break the event $\mathrm{C}_{k}$ (resp. $\mathrm{D}_{k}$ ) into two subevents $\mathrm{C}_{k, c}$ and $\mathrm{C}_{k, s}$ (resp. $\mathrm{D}_{k, c}$ and $\mathrm{D}_{k, s}$ ) as follows:

$$
\begin{aligned}
\mathrm{C}_{k, c}:\left(I_{k+1}=I_{k}\right) & \mathrm{C}_{k, s}:\left(s_{I_{k}}^{k+1} \neq s_{I_{k}}^{k}\right) \\
\mathrm{D}_{k, c}:\left(I_{k}, J_{k}\right)=\left(I_{0}, J_{0}\right) & \mathrm{D}_{k, s}:\left(\wedge_{\ell:=0,2, \ldots, k-2} s_{J_{0}}^{k} \neq s_{J_{0}}^{\ell}\right)
\end{aligned}
$$

The GMF Algorithm is successful in the event $E: B \wedge\left(C_{0} \wedge C_{2} \wedge \cdots \wedge C_{n-1}\right) \wedge\left(D_{2} \wedge D_{4} \wedge \cdots \wedge D_{n-1}\right)$. In other words, with the probability calculated over the randomness of the algorithm GMF Algorithm, it follows that $m f r k_{2}(x)=\operatorname{Pr}[E]$. The task of bounding this probability is accomplished through three claims (Claim 1, Claim 5 and Claim 6). We reuse the bound on $f r k(x)$ given in Claim 1. In order to establish Claim 5 and Claim 6 we have to define a random variable $Z_{i, j}$ that captures a single invocation of the logical wrapper $\mathcal{Z}$. Let $Z_{i, j}: \mathbb{R} \times \mathbb{S}^{j-1} \mapsto[0,1]$, for $1 \leq j \leq q-1$ and $j<i \leq q$, be defined by setting

$$
Z_{i, j}\left(\rho, \mathrm{S}_{(j-)}\right)=\operatorname{Pr}\left[\left(J^{\prime}=J=j\right) \wedge\left(I^{\prime}=I=i\right) \wedge\left(s_{I}^{\prime} \neq s_{I}\right)\right]
$$

given

$$
\begin{gathered}
\left(\mathrm{S}_{(j i)},\left(\mathrm{S}_{(i+)}, \mathrm{S}_{(i+)}^{\prime}\right)\right) \stackrel{\amalg}{\leftarrow} \mathbb{S} \text { and } \\
\left.(I, J, \sigma),\left(I^{\prime}, J^{\prime}, \sigma^{\prime}\right)\right) \leftarrow \mathcal{Z}\left(x,\left\{\mathrm{~S}_{(j-)}, \mathrm{S}_{(j i)}, \mathrm{S}_{(i+)}\right\},\left\{\mathrm{S}_{(j-)}, \mathrm{S}_{(j i)}, \mathrm{S}_{(i+)}^{\prime}\right\} ; \rho\right) .
\end{gathered}
$$

Briefly, our aim is to bound $m f r k k_{2}(x)$ in terms of $Z_{i, j}$ (Claim 5) and bound $Z_{i, j}$ in terms of $f r k(x)$ (Claim 6).

\section{Claim 5.}

$$
m f r k_{2}(x) \geq \frac{1}{q^{n-1}}\left(\sum_{j=1}^{q-1} \sum_{i=j+1}^{q} \operatorname{Ex}\left[Z_{i, j}\right]\right)^{(n+1) / 2}-\frac{(n-1)(n+1)}{8|\mathbb{S}|}\left(\sum_{j=1}^{q-1} \sum_{i=j+1}^{q} \operatorname{Ex}\left[Z_{i, j}\right]\right)
$$

Argument. The first step is to separate the "core" subevents out of the event E as shown below.

$$
\begin{aligned}
\operatorname{Pr}[\mathrm{E}] & =\operatorname{Pr}\left[\mathrm{B} \wedge\left(\wedge_{k=0,2, \ldots, n-1} \mathrm{C}_{k}\right) \wedge\left(\wedge_{k=2,4, \ldots, n-1} \mathrm{D}_{k}\right)\right] \\
& =\operatorname{Pr}\left[\mathrm{B} \wedge \mathrm{C}_{0} \wedge\left(\wedge_{k=2,4, \ldots, n-1} \mathrm{C}_{k} \wedge \mathrm{D}_{k, c} \wedge \mathrm{D}_{k, s}\right)\right] \text { (using subevents given in (33)) } \\
& =\operatorname{Pr}\left[\mathrm{B} \wedge \mathrm{C}_{0} \wedge\left(\wedge_{k=2,4, \ldots, n-1} \mathrm{C}_{k} \wedge \mathrm{D}_{k, c}\right) \wedge\left(\wedge_{k=2,4, \ldots, n-1} \mathrm{D}_{k, s}\right)\right] \\
& \geq \operatorname{Pr}\left[\mathrm{B} \wedge \mathrm{C}_{0} \wedge\left(\wedge_{k=2,4, \ldots, n-1} \mathrm{C}_{k} \wedge \mathrm{D}_{k, c}\right)\right]-\operatorname{Pr}\left[\mathrm{B} \wedge \mathrm{C}_{0} \wedge\left(\vee_{k=2,4, \ldots, n-1} \mathrm{D}_{k, s}\right)\right]
\end{aligned}
$$

We denote the first part of (42) by $m f r k_{2, c}(x)$ and the second part by $m f r k_{2, s}(x)$ and analyse 
them separately.

$$
\begin{aligned}
& m \operatorname{frk}_{2, c}(x)=\operatorname{Pr}\left[\mathrm{B} \wedge \mathrm{C}_{0} \wedge\left(\wedge_{k=2,4, \ldots, n-1} \mathrm{C}_{k} \wedge \mathrm{D}_{k, c}\right)\right] \\
& =\operatorname{Pr}\left[\mathrm{B} \wedge\left(\wedge_{k=0,2, \ldots, n-1} \mathrm{C}_{k}\right) \wedge\left(\wedge_{k=2,4, \ldots, n-1} \mathrm{D}_{k, c}\right)\right] \\
& =\operatorname{Pr}\left[\left(1 \leq J_{0}<I_{0} \leq q\right) \wedge\left(\wedge_{k=0,2, \ldots, n-1}\left(I_{k+1}=I_{k}\right) \wedge\left(s_{I_{k}}^{k+1} \neq s_{I_{k}}^{k}\right)\right) \wedge\right. \\
& \left.\left(\wedge_{k=2,4, \ldots, n-1}\left(I_{k}, J_{k}\right)=\left(I_{0}, J_{0}\right)\right)\right] \\
& =\operatorname{Pr}\left[\left(1 \leq J_{0}<I_{0} \leq q\right) \wedge\left(\wedge_{k=0,2, \ldots, n-1}\left(I_{k+1}, J_{k+1}\right)=\left(I_{k}, J_{k}\right) \wedge\left(s_{I_{k}}^{k+1} \neq s_{I_{k}}^{k}\right)\right) \wedge\right. \\
& \left.\left.\left(\wedge_{k=2,4, \ldots, n-1}\left(I_{k}, J_{k}\right)=\left(I_{0}, J_{0}\right)\right)\right] \text { (since } J \prec I\right) \\
& \left.=\sum_{j=1}^{q-1} \sum_{i=j+1}^{q} \operatorname{Pr}\left[\wedge_{k=0,2, \ldots, n-1}\left(I_{k+1}, J_{k+1}\right)=\left(I_{k}, J_{k}\right)=(i, j) \wedge\left(s_{I_{k}}^{k+1} \neq s_{I_{k}}^{k}\right)\right)\right] \\
& =\sum_{j=1}^{q-1} \sum_{i=j+1}^{q} \sum_{\rho, \mathbf{S}_{(j-)}} \frac{\operatorname{Pr}\left[\wedge_{k=0,2, \ldots, n-1}\left(I_{k+1}, J_{k+1}\right)=\left(I_{k}, J_{k}\right)=(i, j) \wedge\left(s_{I_{k}}^{k+1} \neq s_{I_{k}}^{k}\right)\right]}{|\mathbb{R}||\mathbb{S}|^{j-1}} \\
& =\sum_{j=1}^{q-1} \sum_{i=j+1}^{q} \sum_{\rho, \mathrm{S}_{(j-)}} \frac{\prod_{k=0,2}^{n-1} \operatorname{Pr}\left[\left(I_{k+1}, J_{k+1}\right)=\left(I_{k}, J_{k}\right)=(i, j) \wedge\left(s_{I_{k}}^{k+1} \neq s_{I_{k}}^{k}\right) \mid \bigwedge_{l=0,2}^{k-2} \mathrm{G}_{\ell}\right]}{|\mathbb{R} \| \mathbb{S}|^{j-1}}
\end{aligned}
$$

In the above expression, $\mathrm{G}_{\ell}$ denotes the event

$$
\left(I_{\ell+1}, J_{\ell+1}\right)=\left(I_{\ell}, J_{\ell}\right)=(i, j) \wedge\left(s_{I_{\ell}}^{\ell+1} \neq s_{I_{\ell}}^{\ell}\right) .
$$

Using the random variable $Z_{i, j}$, (43) can be rewritten as

$$
\begin{aligned}
m f r k_{2, c}(x) & =\sum_{j=1}^{q-1} \sum_{i=j+1}^{q} \sum_{\rho, \mathrm{S}_{(j-)}} \frac{\prod_{k=0,2}^{n-1} Z_{i, j}\left(\rho, \mathrm{S}_{(j-)}\right)}{|\mathbb{R}||\mathbb{S}|^{j-1}} \\
& =\sum_{j=1}^{q-1} \sum_{i=j+1}^{q} \sum_{\rho, \mathrm{S}} \frac{Z_{(j-)}^{(n+1) / 2}\left(\rho, \mathrm{S}_{(j-)}\right)}{|\mathbb{R}||\mathbb{S}|^{j-1}} \\
& =\sum_{j=1}^{q-1} \sum_{i=j+1}^{q} \operatorname{Ex}\left[Z_{i, j}^{(n+1) / 2}\right] \geq \sum_{j=1}^{q-1} \sum_{i=j+1}^{q} \operatorname{Ex}\left[Z_{i, j}\right]^{(n+1) / 2} \quad \text { (by Jensen's inequality) } \\
& \geq \frac{1}{q^{n-1}}\left(\sum_{j=1}^{q-1} \sum_{i=j+1}^{q} \operatorname{Ex}\left[Z_{i, j}\right]\right)^{(n+1) / 2} \quad \text { (by Hölder's inequality) }
\end{aligned}
$$

Using a similar line of approach (as in (43) and (44)), it is possible to establish that

$$
m f r k_{2, s}(x)=\frac{(n-1)(n+1)}{8|\mathbb{S}|}\left(\sum_{j=1}^{q-1} \sum_{i=j+1}^{q} \operatorname{Ex}\left[Z_{i, j}\right]\right) .
$$

Substituting the value of $m f r k_{2, c}(x)$ from (44) and $m f r k_{2, s}(x)$ from (45) in (42), yields the bound in Claim 5.

What remains is to relate Claim 1 and Claim 5

Claim 6. $\sum_{j=1}^{q-1} \sum_{i=j+1}^{q} \operatorname{Ex}\left[Z_{i, j}\right] \geq \operatorname{frk}(x)$. 
Argument. This argument makes use of the partitions of $\mathbb{T}_{(2)}$ that we used in Claim 3 . Let $\mathbb{T}_{(2, j i)}$ denote the underlying set for the random variable $Z_{i, j}$. From the definition of $Z_{i, j}$, we can infer that

$$
\begin{gathered}
\mathbb{T}_{(2, j i)}:=\left(\mathbb{R} \times \mathbb{S}^{j-1} \times \mathbb{S}^{i-j} \times \mathbb{S}^{(q-i+1) * 2}\right)=\left(\mathbb{R} \times \mathbb{S}^{i-1} \times \mathbb{S}^{(q-i+1) * 2}\right) \text { and } \\
\operatorname{Ex}\left[Z_{i, j}\right]=\operatorname{T}_{(2, j i)}\left[\left(J^{\prime}=J=j\right) \wedge\left(I^{\prime}=I=i\right) \wedge\left(s_{I}^{\prime} \neq s_{I}\right)\right] .
\end{gathered}
$$

Notice that $\mathbb{T}_{(2, j i)}$ is the subset of $\mathbb{T}_{(2)}$. Let $\overline{\mathbb{T}}_{(2, j i)}$ denote $\mathbb{T}_{(2)} \backslash \mathbb{T}_{(2, j i)}$. It follows by definition that

$$
\underset{\overline{\mathbb{T}}_{(2, j i)}}{\operatorname{Pr}}\left[\left(J^{\prime}=J=j\right) \wedge\left(I^{\prime}=I=i\right) \wedge\left(s_{I}^{\prime} \neq s_{I}\right)\right]=0 .
$$

The fact that $\mathbb{T}_{(2, j i)} \subset \mathbb{T}_{(2)}$ and (46) implies

$$
\begin{gathered}
\operatorname{Pr}_{(2, j i)}\left[\left(J^{\prime}=J=j\right) \wedge\left(I^{\prime}=I=i\right) \wedge(j<I \leq q) \wedge\left(s_{I}^{\prime} \neq s_{I}\right)\right] \geq \\
\underset{\mathbb{T}_{(2)}}{\operatorname{Pr}}\left[\left(J^{\prime}=J=j\right) \wedge\left(I^{\prime}=I=i\right) \wedge(j<I \leq q) \wedge\left(s_{I}^{\prime} \neq s_{I}\right)\right] .
\end{gathered}
$$

On taking the sum of $\operatorname{Ex}\left[Z_{i, j}\right]$ over the indices $(i, j)$, we get

$$
\begin{aligned}
\sum_{j=1}^{q-1} \sum_{i=j+1}^{q} \operatorname{Ex}\left[Z_{i, j}\right] & \geq \sum_{j=1}^{q-1} \sum_{i=j+1}^{q} \underset{\mathbb{T}_{(2)}}{\operatorname{Pr}}\left[\left(J^{\prime}=J=j\right) \wedge\left(I^{\prime}=I=i\right) \wedge(j<I \leq q) \wedge\left(s_{I} \neq s_{I}^{\prime}\right)\right] \\
& =\underset{\mathbb{T}_{(2)}}{\operatorname{Pr}}\left[\left(J^{\prime}=J\right) \wedge\left(I^{\prime}=I\right) \wedge(1 \leq J<I \leq q) \wedge\left(s_{I} \neq s_{I}^{\prime}\right)\right]=f r k(x)
\end{aligned}
$$

completing the argument.

On putting all the three claims together, we get

$$
\begin{aligned}
\operatorname{mfrk}_{2}(x) & \geq \frac{1}{q^{n-1}} \operatorname{frk}(x)^{(n+1) / 2}-\frac{(n-1)(n+1)}{8|\mathbb{S}|} \operatorname{frk}(x) \\
& =f r k(x) \cdot\left(\frac{\operatorname{frk}(x)^{(n-1) / 2}}{q^{n-1}}-\frac{(n-1)(n+1)}{8|\mathbb{S}|}\right)
\end{aligned}
$$

Finally, taking the expectation over $x \stackrel{\$}{\leftarrow} \mathcal{G}_{I}$, yields

$$
f r k \geq \operatorname{acc}\left(\frac{\operatorname{acc}}{q}(1-\eta)-\frac{1}{|\mathbb{S}|}\right) \text { and } m f r k_{2} \geq f r k \cdot\left(\frac{f r k^{(n-1) / 2}}{q^{n-1}}-\frac{(n-1)(n+1)}{8|\mathbb{S}|}\right)
$$

establishing Lemma 10. We conclude with the comment that on assuming $|\mathbb{S}| \gg 1$, one gets $m f r k_{2} \approx a c c^{n+1} / q^{(3 n-1) / 2}$.

\section{Constructions}

\section{D.1 The Boldyreva-Palacio-Warinschi Proxy Signature Scheme}

Let $\mathfrak{S}=\left\{\mathcal{G}_{s}, \mathcal{K}_{s}, \mathcal{S}_{s}, \mathcal{V}_{s}\right\}$ denote the Schnorr signature scheme. The Triple-Schnorr proxy signature scheme $\mathfrak{B}$ consists of the algorithms $\left(\mathcal{G}, \mathcal{K}, \mathcal{S}, \mathcal{V},(\mathcal{D}, \mathcal{P}), \mathcal{S}_{p}, \mathcal{V}, \mathcal{I}\right)$, each of which is defined below. 
Parameter Generation, $\mathcal{G}(\kappa)$ : Run the Schnorr Parameter Generation algorithm $\mathcal{G}_{s}$ on $\kappa$ to obtain $\mathrm{pp}_{s}=(\mathbb{G}, g, p, \mathrm{G})$. Return $\mathrm{pp}:=\left(\mathrm{pp}_{s}, \mathrm{H}, \mathrm{R}\right)$ as public parameters, where $\mathrm{H}$ and $\mathrm{R}$ are two hash functions

$$
\mathrm{H}:\{0,1\}^{*} \mapsto \mathbb{Z}_{p} \text { and } \mathrm{R}:\{0,1\}^{*} \mapsto \mathbb{Z}_{p}
$$

Key Generation, $\mathcal{K}(\mathrm{pp})$ : Run the Schnorr Key Generation algorithm $\mathcal{K}_{s}$ on $\mathrm{pp}_{s}$ to obtain $\left(\left(\mathrm{pp}_{s}, X\right),\left(\mathrm{pp}_{s}, x\right)\right)$. Return $\mathrm{pk}:=(\mathrm{pp}, X)$ as the public key and $\mathrm{sk}:=(\mathrm{pp}, x)$ as the secret key. For convenience, we drop pp from the keys; thus, $(X, x)$ serves as the key-pair.

Signing, $\mathcal{S}(m, \mathrm{sk})$ : Return $\mathcal{S}_{s}^{\mathrm{G}}(1 \| m, \mathrm{sk})$ as the signature on the message $m$.

Verification, $\mathcal{V}(m, \sigma, \mathrm{pk})$ : $\operatorname{Return} \mathcal{V}_{s}^{\mathrm{G}}(\sigma, 1 \| m, \mathrm{pk})$.

Delegation, $\left(\mathcal{D}\left(\mathrm{pk}_{i}, \mathrm{sk}_{i}, j, \mathrm{pk}_{j}, \tilde{\mathrm{M}}\right), \mathcal{P}\left(\mathrm{pk}_{j}, \mathrm{sk}_{j}, \mathrm{pk}_{i}\right)\right)$ : Let $S$ be a shorthand for the string $\left(\mathrm{pk}_{i}\|j\| \mathrm{pk}_{j} \| \tilde{\mathbb{M}}\right)$. User $i$ computes

$$
\text { cert }:=(Y, s) \stackrel{\$}{\leftarrow} \mathcal{S}_{s}^{\mathrm{G}}\left(\mathrm{sk}_{i}, 0 \| S\right)
$$

and sends it to user $j$. User $j$, in turn, verifies cert and computes the proxy signing key $\operatorname{skp}:=(S, Y, t)$ where

$$
t=r \cdot \mathrm{sk}_{j}+s \text { with } r:=\mathrm{R}(S\|Y\| c) \text { and } c:=\mathrm{G}(0\|S\| Y) .
$$

Proxy Signing, $\mathcal{S}_{\underline{p}}(\operatorname{skp}, \tilde{m})$ : Let $r:=\mathrm{R}(S\|Y\| c)$ and $c:=\mathrm{G}(0\|S\| Y)$. If $\tilde{m}$ is indeed from the message space $\tilde{\mathbb{M}}$ then return

$$
\sigma_{p}:=\left(j, \mathrm{pk}_{j}, \tilde{\mathbb{M}}, Y, \mathcal{S}_{s}^{\mathrm{H}}(t, 0\|\tilde{m}\| S\|Y\| r)\right)
$$

as the proxy signature on $\tilde{m}$ on behalf of user $j$ by the user $i$. Else, return $\perp$.

Proxy Verification, $\mathcal{V}_{p}\left(\sigma_{p}, \tilde{m}, \mathrm{pk}_{i}\right)$ : Let $\sigma_{p}$ be of the form $\left(j, \mathrm{pk}_{j}, \tilde{\mathbb{M}}, Y, \sigma\right)$. Compute the stamp $S:=\left(\mathrm{pk}_{i}\|j\| \mathrm{pk}_{j} \| \tilde{\mathrm{M}}\right)$. In addition, compute the proxy public key

$$
\operatorname{pkp}:=\mathrm{pk}_{j}^{r} \cdot Y \cdot \mathrm{pk}_{i}^{c}
$$

where $r:=\mathrm{R}(S\|Y\| c)$ and $c:=\mathrm{G}(0\|S\| Y)$. Return $\mathcal{V}_{s}^{\mathrm{H}}(\sigma, 0\|\tilde{m}\| S$, pkp $)$

Proxy Identification, $\mathcal{I}\left(\sigma_{p}\right)$ : Let $\sigma_{p}$ be of the form $\left(j, \mathrm{pk}_{j}, \tilde{\mathbb{M}}, Y, \sigma\right)$. Return $j$ as the identity of the proxy signer.

Figure 5: The BPW Proxy Signature Scheme. (We use $\tilde{\mathbb{M}}$ in place of $\omega$ (used in [BPW12]) to maintain uniformity of notation.)

Remark 8. Self-delegation can be achieved by invoking the interactive algorithm $(\mathcal{D}, \mathcal{P})$ on an alternative key-pair of the designator (itself), in place of the key-pair of the proxy signer. For example, a user $i$ with an alternative key-pair $\left(\mathrm{pk}_{i}^{\prime}, \mathrm{sk}_{i}^{\prime}\right)$ can delegate itself by invoking

$$
(\mathrm{skp}, \perp) \stackrel{\$}{\leftarrow}\left(\mathcal{D}\left(\mathrm{pk}_{i}, \mathrm{sk}_{i}, i, \mathrm{pk}_{i}^{\prime}, \tilde{\mathrm{M}}\right), \mathcal{P}\left(\mathrm{pk}_{i}^{\prime}, \mathrm{sk}_{i}^{\prime}, \mathrm{pk}_{i}\right)\right)
$$




\section{D.2 The (Original) Galindo Garcia IBS}

Set-up, $\mathcal{G}(\kappa)$ : Invoke the group generator $\mathcal{G}_{\mathrm{DL}}$ (on $1^{\kappa}$ ) to obtain $(\mathbb{G}, g, p)$. Return $z \stackrel{U}{\leftarrow} \mathbb{Z}_{p}$ as the master secret key msk and $\left(\mathbb{G}, p, g, g^{z}, \mathrm{H}, \mathrm{G}\right)$ as the master public key mpk, where $\mathrm{H}$ and $\mathrm{G}$ are hash functions

$$
\mathrm{H}:\{0,1\}^{*} \mapsto \mathbb{Z}_{p} \text { and } \mathrm{G}:\{0,1\}^{*} \mapsto \mathbb{Z}_{p}
$$

Key Extraction, $\mathcal{E}$ (id,msk, mpk): Select $r \stackrel{\cup}{\leftarrow} \mathbb{Z}_{p}$ and set $R:=g^{r}$. Return usk $:=(y, R) \in$ $\mathbb{Z}_{p} \times \mathbb{G}$ as the user secret key, where

$$
y:=r+z c \text { and } c:=\mathrm{H}(R \| \mathrm{id}) .
$$

Signing, $\mathcal{S}$ (id, $m$, usk, mpk): Let usk $=(y, R)$ and $c=\mathrm{H}(R \|$ id $)$. Select $a \stackrel{\mathrm{U}}{\leftarrow} \mathbb{Z}_{p}$ and set $A:=g^{a}$. Return $\sigma:=(b, R, A) \in \mathbb{G} \times \mathbb{Z}_{p} \times \mathbb{G}$ as the signature, where

$$
b:=a+y d \text { and } d:=\mathrm{G}(\mathrm{id}\|A\| m) .
$$

Verification, $\mathcal{V}(\sigma, \mathrm{id}, m, \mathrm{mpk})$ : Let $\sigma=(b, R, A), c:=\mathrm{H}(R \|$ id $)$ and $d:=\mathrm{G}(\mathrm{id}\|A\| m)$. The signature is valid if

$$
g^{b}=A\left(R \cdot\left(g^{z}\right)^{c}\right)^{d} .
$$

Figure 6: The (Original) Galindo-Garcia IBS.

\section{D.3 The Modified Galindo Garcia IBS}

The construction is same as in Figure 6 except for the structure of the hash functions. We have introduced a binding between $\mathrm{H}$ and $\mathrm{G}$ (through $d:=\mathrm{G}(m, A, c)$ where $c:=\mathrm{H}(\mathrm{id}, R)$ ) to induce a dependence of $\mathrm{H} \prec \mathrm{G}$. The binding that we have introduced is more refined than the one suggested in $\S 4.3 .1$ (i.e., $d:=\mathrm{G}(\mathrm{id}\|A\| m \| c)$ where $c:=\mathrm{H}(R \|$ id $)$ ).

Set-up, $\mathcal{G}(\kappa)$ : Generate a group $\mathbb{G}=\langle g\rangle$ of prime order $p$. Return $z \stackrel{\mathrm{U}}{\leftarrow} \mathbb{Z}_{p}$ as the master secret key msk and $\left(\mathbb{G}, p, g, g^{z}, \mathrm{H}, \mathrm{G}\right)$ as the master public key mpk, where $\mathrm{H}$ and $\mathrm{G}$ are hash functions

$$
\mathrm{H}:\{0,1\}^{*} \times \mathbb{G} \mapsto \mathbb{Z}_{p} \text { and } \mathrm{G}:\{0,1\}^{*} \times \mathbb{G} \times \mathbb{Z}_{p} \mapsto \mathbb{Z}_{p}
$$

Key Extraction, $\mathcal{E}$ (id,msk, mpk): Select $r \stackrel{\cup}{\leftarrow} \mathbb{Z}_{p}$ and set $R:=g^{r}$. Return usk $:=(y, R) \in$ $\mathbb{Z}_{p} \times \mathbb{G}$ as the user secret key, where

$$
y:=r+z c \text { and } c:=\mathrm{H}(\mathrm{id}, R) .
$$

Signing, $\mathcal{S}$ (id, $m$, usk, mpk): Let usk $=(y, R)$ and $c=\mathrm{H}(\mathrm{id}, R)$. Select $a \stackrel{\mathrm{U}}{\leftarrow} \mathbb{Z}_{p}$ and set $A:=g^{a}$. Return $\sigma:=(b, R, A) \in \mathbb{G} \times \mathbb{Z}_{p} \times \mathbb{G}$ as the signature, where

$$
b:=a+y d \text { and } d:=\mathrm{G}(m, A, c) .
$$


Verification, $\mathcal{V}(\sigma, \mathrm{id}, m, \mathrm{mpk})$ : Let $\sigma=(b, R, A), c:=\mathrm{H}(\mathrm{id}, R)$ and $d:=\mathrm{G}(m, A, c)$. The signature is valid if

$$
g^{b}=A\left(R \cdot\left(g^{z}\right)^{c}\right)^{d} .
$$

Figure 7: The Modified Galindo-Garcia IBS.

\section{D.4 Chow-Ma-Weng Zero-Knowledge Argument}

Chaum and Pederson devised a zero-knowledge protocol for proving the equality of two discrete logarithms [CP93]. The protocol due to Chow et al. improves on that of Chaum and Pederson by increasing efficiency and, also, by including provision for simultaneous checking of $n \geq 2$ discrete logarithms.

Setting. Let $\mathbb{G}$ be a cyclic group of order a prime $p$. Let $g, h \in \mathbb{G}$ be two random generators for $\mathbb{G}$. In addition, let $\mathrm{H}: \mathbb{G}^{2} \mapsto \mathbb{Z}_{p}^{*}$ be a cryptographic hash function.

Protocol. The objective of the prover $\mathcal{P}$, after publishing $y_{1}=g^{x}$ and $y_{2}=h^{x}$, is to convince the verifier $\mathcal{V}$ that $\log _{g} y_{1}=\log _{h} y_{2}$. This is accomplished through the following sequence of steps:

(i) $\mathcal{P}$ picks $k \stackrel{\cup}{\leftarrow} \mathbb{Z}_{p}^{*}$ and sends the commitment $v:=\left(g^{z} h\right)^{k}$ to $\mathcal{V}$, where $z:=\mathrm{H}\left(y_{1}, y_{2}\right)$.

(ii) Upon receiving $v, \mathcal{V}$ sends challenge $c \stackrel{\cup}{\leftarrow} \mathbb{Z}_{p}^{*}$ to $\mathcal{P}$.

(iii) $\mathcal{P}$ sends the response $s:=k-c x \bmod p$ to $\mathcal{V}$.

(iv) $\mathcal{V}$ accepts iff $v=\left(g^{z} h\right)^{s}\left(y_{1}^{z} y_{2}\right)^{c}$ holds, where $z:=\mathrm{H}\left(y_{1}, y_{2}\right)$.

Figure 8: The Chow-Ma-Weng argument for the statement $\log _{g} y_{1}=\log _{g} y_{2}$.

\section{E Security Argument for Modified Galindo-Garcia IBS}

Theorem 1. Let $\mathcal{A}$ be an $\left(\epsilon, t, q_{\varepsilon}, q_{s}, q_{H}, q_{G}\right)$-adversary against the modified GG-IBS. If $H$ and $G$ are modelled as random oracles, we can construct either

(i) Algorithm $\mathcal{R}_{1}^{\prime}$ which $\epsilon_{1}$-breaks the $D L P$, where $\epsilon_{1}=O\left(\epsilon^{2} /\left(\exp (1) q_{G} q_{\varepsilon}\right)\right)$ or

(ii) Algorithm $\mathcal{R}_{3}^{\prime}$ which $\epsilon_{3}$-breaks the DLP, where $\epsilon_{3}=O\left(\epsilon^{4} /\left(q_{H}+q_{G}\right)^{3}\right)$.

Here $q_{\varepsilon}$ and $q_{s}$ denote the upper bound on the number of extract and signature queries, respectively, that $\mathcal{A}$ can make; $q_{H}$ and $q_{G}$ denote the upper bound on the number of queries to the H-oracle and G-oracle respectively.

Argument. $\mathcal{A}$ is successful if it produces a valid non-trivial forgery $\hat{\sigma}=(\hat{b}, \hat{R}, \hat{A})$ on $(\hat{i d}, \hat{m})$. Consider the following complementary events in the case that $\mathcal{A}$ is successful.

$\mathrm{E}: \mathcal{A}$ makes at least one signature query on $\hat{i d}$ and $\hat{R}$ was returned by the simulator as part of the output to a signature query on $\hat{i d}$.

$\overline{\mathrm{E}}$ : Either $\mathcal{A}$ does not make any signature queries on $\hat{i d}$ or $\hat{R}$ was never returned by the simulator as part of the output to a signature query on $\hat{i d}$. 
In the event $\mathrm{E}$ we give a reduction $\mathcal{R}_{1}^{\prime}$, whereas in the event $\overline{\mathrm{E}}$, we give $\mathcal{R}_{3}^{\prime}$. Apart from the need of a wrapper, $\mathcal{R}_{1}^{\prime}$ is similar to the reduction $\mathcal{R}_{1}$ given in [CKK13]. $\mathcal{R}_{3}^{\prime}$, on the other hand, employs the GMF Algorithm $\mathcal{N}_{\mathbb{A}_{3}, \mathcal{Y}, 3}$ (with Lemma 2), in place of $\mathcal{M}_{\mathcal{Y}, 3}$. Hence, we confine the security argument to the details of reduction $\mathcal{R}_{3}^{\prime}$.

Remark 9. The dependence among the hash functions H and G (modelled as random oracles) has been induced by the binding between them. The notion of independence, on the other hand, can be argued in terms of the system of congruences as we have done for BPW-PSS and CMW-ZKP.

\section{E.1 Reduction $\mathcal{R}_{3}^{\prime}$}

Let $\Delta:=\left(\mathbb{G}, p, g, g^{\alpha}\right)$ be the given DLP instance. The reduction involves invoking the GMF Algorithm $\mathcal{N}_{\mathbb{A}_{3}, \mathcal{Y}, n}$ on the wrapper $\mathcal{Y}$ as shown in Algorithm 5. As a result, it obtains a set of four congruences in four unknowns and solves for $\alpha$. It can be verified that $\mathcal{R}_{3}^{\prime}$ indeed returns the correct solution to the DLP instance (see full version of [CKK13] for details). The design of the wrapper $\mathcal{Y}$ follows.

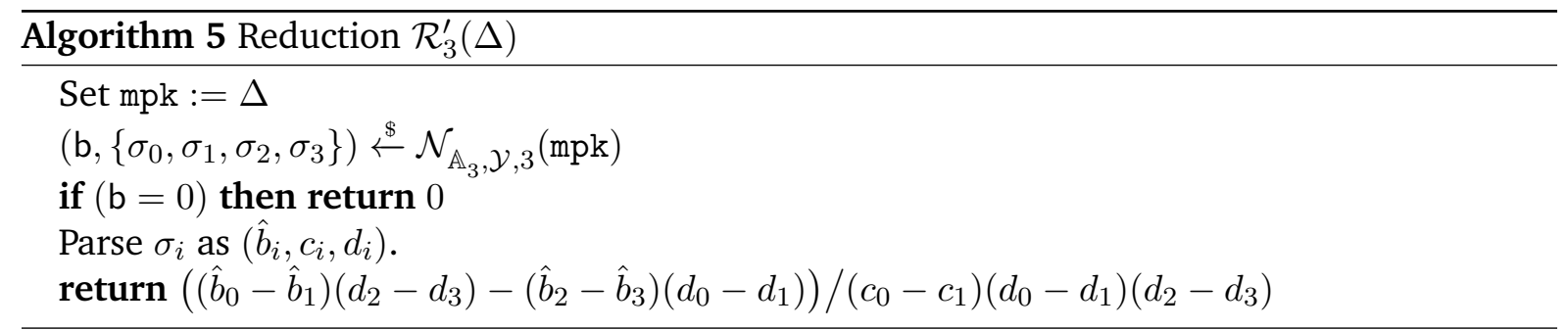

\section{The Wrapper}

Suppose that $q:=q_{\mathrm{H}}+q_{\mathrm{G}}$ and $\mathbb{S}:=\mathbb{Z}_{p} . \mathcal{Y}$ takes as input the master public key mpk and $s_{1}, \ldots, s_{q}$, and returns a triple $(I, J, \sigma)$ where $J$ and $I$ are integers that refer to the target $\mathrm{H}$ and G query respectively and $\sigma$ is the side-output. In order to track the index of the current random oracle query, $\mathcal{Y}$ maintains a counter $\ell$, initially set to 1 . It also maintains a table $\mathfrak{L}_{\mathrm{H}}$ (resp. $\mathfrak{L}_{\mathrm{G}}$ ) to manage the random oracle $\mathrm{H}$ (resp. G). $\mathcal{Y}$ initiates the simulation of the protocol environment by passing mpk as the challenge master public key to the adversary $\mathcal{A}$. The queries by $\mathcal{A}$ are handled as per the following specifications.

(a) H-oracle Query. $\mathfrak{L}_{\mathrm{H}}$ contains tuples of the form $\langle\mathrm{id}, R, c, \ell, y\rangle$. Here, (id, $R$ ) is the query to the $\mathrm{H}$-oracle with $c$ being the corresponding output. The index of the oracle call is stored in the $\ell$-field. Finally, the $y$-field stores either (a component of) the secret key for id, or a ' $\perp$ ' in case the field is invalid. A fresh H-oracle query is handled as follows: i) return $c:=s_{\ell}$ as the output; and ii) add $\langle i d, R, c, \ell, \perp\rangle$ to $\mathfrak{L}_{\mathrm{H}}$ and increment $\ell$ by one.

(b) G-oracle Query. $\mathfrak{L}_{\mathrm{G}}$ contains tuples of the form $\langle m, A, c, d, \ell\rangle$. Here, $(m, A, c)$ is the query to the G-oracle with $d$ being the corresponding output. The index of the oracle call is stored in the $\ell$-field. A fresh G-oracle query is handled as follows: i) return $d:=s_{\ell}$ as the output; and ii) add $\langle m, A, c, d, \ell\rangle$ to $\mathfrak{L}_{\mathrm{G}}$ and increment $\ell$ by one.

(c) Signature and Extract Queries. Since the master secret key $\alpha$ is unknown to $\mathcal{Y}$, it has to carefully program the $\mathrm{H}$-oracle in order to generate the user secret key usk. The signature queries, on the other hand, are answered by first generating the usk (as in the extract query), followed by invoking $\mathcal{S}$.

Extract query. $\mathcal{O}_{\varepsilon}(\mathrm{id})$ : 
(i) If there exists a tuple $\left\langle\mathrm{id}_{i}, R_{i}, c_{i}, \ell_{i}, y_{i}\right\rangle$ in $\mathfrak{L}_{\mathrm{H}}$ such that $(\mathrm{id} i=\mathrm{id}) \wedge\left(y_{i} \neq \perp\right), \mathcal{Y}$ returns usk : $=\left(y_{i}, R_{i}\right)$ as the secret key.

(ii) Otherwise, $\mathcal{Y}$ chooses $y \stackrel{U}{\leftarrow} \mathbb{Z}_{p}$, sets $c:=s_{\ell}$ and $R:=\left(g^{\alpha}\right)^{-c} g^{y}$. It then adds $\langle\text { id, } R, c, \ell, y\rangle^{21}$ to $\mathfrak{L}_{\mathrm{H}}$ and increments $\ell$ by one (an implicit H-oracle call). Finally, it returns usk $:=(y, R)$ as the secret key.

Signature query. $\mathcal{O}_{s}(\mathrm{id}, m)$ :

(i) If there exists a tuple $\left\langle\mathrm{id}_{i}, R_{i}, c_{i}, \ell_{i}, y_{i}\right\rangle$ in $\mathfrak{L}_{\mathrm{H}}$ such that $\left(\mathrm{id}_{i}=\mathrm{id}\right) \wedge\left(y_{i} \neq \perp\right)$, then usk $=\left(y_{i}, R_{i}\right)$. $\mathcal{Y}$ now uses the knowledge of usk to run $\mathcal{S}$ and returns the signature.

(ii) Otherwise, $\mathcal{Y}$ generates the usk as in step (ii) of Extract query and runs $\mathcal{S}$ to return the signature.

The Output. At the end of the simulation, a successful adversary outputs a valid forgery $\hat{\sigma}:=$ $(\hat{b}, \hat{R}, \hat{A})$ on a $(\hat{\mathrm{id}}, \hat{m})$. Let $\left\langle\mathrm{id}_{j}, R_{j}, c_{j}, \ell_{j}, y_{j}\right\rangle$ be the tuple in $\mathfrak{L}_{\mathrm{H}}$ that corresponds to the target $\mathrm{H}$ query. Similarly, let $\left\langle m_{i}, A_{i}, c_{i}, d_{i}, \ell_{i}\right\rangle$ be the tuple in $\mathfrak{L}_{\mathrm{G}}$ that corresponds to the target G-query. $\mathcal{Y}$ returns $\left(l_{i}, \ell_{j},\left(\hat{b}, c_{j}, d_{i}\right)\right)$ as its own output. Note that the side-output $\sigma$ consists of $\left(\hat{b}, c_{j}, d_{i}\right)$.

\section{E.1.1 Analysis.}

Since there is no abort involved in the simulation of the protocol, we may conclude that the accepting probability of $\mathcal{Y}$ is the same as the advantage of the adversary, i.e. acc $=\epsilon$. The probability of success of the reduction $\mathcal{R}_{3}^{\prime}$ is computed by using Lemma 2 with $q:=q_{\mathrm{H}}+q_{\mathrm{G}}$, $|\mathbb{S}|:=p$ and $n=3$. Hence, we have $\epsilon_{3}=\mathrm{O}\left(\epsilon^{4} /\left(q_{\mathrm{H}}+q_{\mathrm{G}}\right)^{3}\right)$.

\footnotetext{
${ }^{21}$ In the unlikely event of there already existing a tuple $\left\langle\mathrm{id}_{i}, R_{i}, c_{i}, \ell_{i}, \perp\right\rangle$ in $\mathfrak{L}_{\mathrm{H}}$ with $\left(\mathrm{id}_{i}=\mathrm{id}\right) \wedge\left(R_{i}=R\right) \wedge\left(c_{i}=\right.$ $c), \mathcal{Y}$ will simply increment $\ell$ and repeat step (ii).
} 